Document downloaded from:

http://hdl.handle.net/10251/68257

This paper must be cited as:

Sirci, S.; Sánchez Soriano, MÁ.; Martínez Pérez, JD.; Boria Esbert, VE.; Gentili, F.; Boesch, W.; Sorrentino, R. (2015). Design and Multiphysics Analysis of Direct and Cross-Coupled SIW Combline Filters Using Electric and Magnetic Couplings. IEEE Transactions on Microwave Theory and Techniques. 63(12):4341-4354. doi:10.1109/TMTT.2015.2495287.

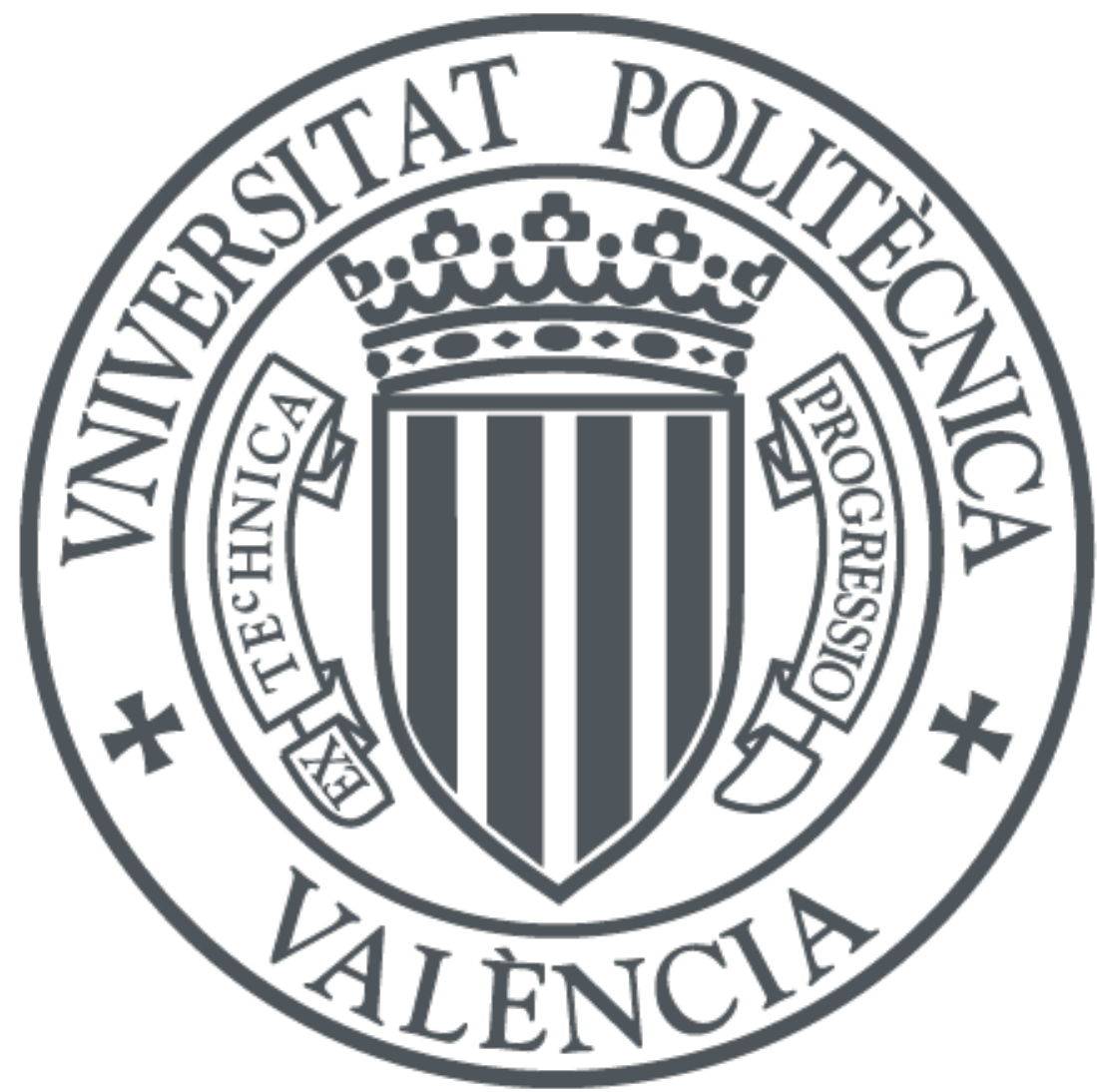

The final publication is available at

http://dx.doi.org/10.1109/TMTT.2015.2495287

Copyright Institute of Electrical and Electronics Engineers (IEEE)

Additional Information 


\title{
Design and Multiphysics Analysis of Direct and Cross-Coupled SIW Combline Filters using Electric and Magnetic Couplings
}

\author{
Stefano Sirci, Student Member, IEEE, Miguel Ángel Sánchez-Soriano, Member, IEEE, \\ Jorge D. Martínez, Member, IEEE, Vicente E. Boria, Senior Member, IEEE, Fabrizio Gentili, Associate \\ Member, IEEE, Wolfgang Bösch, Fellow, IEEE and Roberto Sorrentino, Fellow, IEEE
}

\begin{abstract}
In this paper, combline substrate integrated waveguide (SIW) filters using electric and magnetic couplings are thoroughly studied. Thus, a negative coupling scheme consisting on an open-ended coplanar probe is proposed and analyzed in detail. Several in-line 3 -pole filters at $\mathrm{C}$-band are designed, manufactured and measured showing how the presented approach can be used for implementing direct couplings while enabling an important size reduction and improved spurious-free band compared to conventional magnetic irises. A fully-packaged quasi-elliptic 4 -pole filter is also designed at $5.75 \mathbf{~ G H z}$ showing how the negative coupling structure can be used for introducing transmission zeros by means of cross-couplings between nonadjacent resonators. Finally, average and peak power handling capabilities of these filters have been also analyzed from a multiphysics point of view. Measured results validate the theoretical predictions confirming that combline SIW filters can handle significant levels of continuous and peak power, providing at the same time easy integration, compact size and advanced filtering responses.
\end{abstract}

Index Terms-Cross-coupled filters, electric and magnetic mixed couplings, multiphysics analysis, power handling capabilities, quasi-elliptic filter, substrate integrated waveguide.

\section{INTRODUCTION}

$\mathbf{S}$ UBSTRATE integrated waveguide (SIW) has already demonstrated to be a successful approach for implementing microwave and $\mathrm{mm}$-wave filters with high Q-factor, easy integration with planar circuits, and mass production manufacturing processes in PCB and LTCC technology [1]. However, other potential features that, combined with the former advantages, could be of huge interest in a wide range of wireless and mobile applications are a lively subject of research, like compactness, advanced filtering responses, and recently power handling capabilities.

Manuscript received July 1, 2015. This work was supported in part by MINECO (Spanish Government) under projects TEC2013-47037-C5-1-R and TEC2013-48036-C3-3-R.

S. Sirci, M.A. Sánchez-Soriano and V.E. Boria are with the iTEAM, Universitat Politècnica de València, Camino de Vera s/n, E-46022, Valencia, Spain (e-mail: ssirci@iteam.upv.es, m.sanchez.soriano@ieee.org, vboria@dcom.upv.es).

J.D. Martínez is with the I3M, Universitat Politècnica de València, Valencia, Spain (e-mail: jdmartinez@eln.upv.es).

F. Gentili and W. Bösch are with the IHF, Graz University of Technology, Graz, Austria (e-mail: fabrizio.gentili@tugraz.at, wbosch@tugraz.at).

R. Sorrentino is with the University of Perugia, Perugia, Italy (e-mail: roberto.sorrentino@unipg.it).
The realization of compact SIW filters has been approached from different perspectives. Several authors have proposed more compact alternatives to conventional SIW cavities by bisecting the resonator at quasi-perfect magnetic walls. Among the most relevant techniques that can be identified in the literature, there are folded [2], half-mode [3] and quartermode [4] SIW bandpass filters. Other approaches have focused on loading the SIW resonator with complementary split-ring resonators [5], dielectric rods [6] and more recently combline SIW filters, which were proposed as a translation of the wellknown 3D coaxial resonator concept to a substrate integrated scheme [7]. Even with some trade-offs in terms of Q-factor or manufacturing complexity, most of the former approaches can obtain significant size reduction, but keeping fabrication and integration easiness (i.e. preferably single-layer batchfabrication processes with solid bottom ground planes) that are usually of major importance from a practical point of view.

At the same time, filtering functions including transmission zeros (TZs) at finite or imaginary frequencies are of great interest in many applications, enabling to achieve higher selectivity with a reduced footprint. In this sense, the use of cross-couplings between resonators is a well-known and extended technique for the introduction of TZs, based on the generation of multiple paths between the filter input and output, and therefore allowing for signal cancellation [8], [9]. Even if positive and negative couplings are generally required, magnetic coupling using irises between adjacent resonators has been the preeminent scheme, and the use of all inductive couplings has been already demonstrated for implementing linear-phase [10] and trisection [11] band-pass SIW filters. On the other hand, electric coupling mechanisms in SIW structures have been investigated by several authors. In [12] and [13], a negative coupling structure between SIW cavity resonators is proposed using a balanced microstrip line with a pair of plated via holes. However, in both structures, slots need to be etched at the top and bottom layers of the substrate, limiting the integration and packaging possibilities of the devices. In [14], a controllable mixed coupling is created using an embedded short-ended stripline combined with a wall iris at the expense of a multi-layer fabrication process that increases the complexity of the structure. On the other hand, grounded coplanar lines [15], microstrip lines [16] and oversized cavities [17] have been proposed as single-layer 
solutions, although some of the former structures are limited in terms of flexibility (i.e. coupling level or resonator positioning) or they require a larger area. Following this approach, the authors have recently proposed a solution for obtaining electric coupling in combline SIW resonators using a single-layer open-ended coplanar probe [18].

Lastly, even if higher power capacity is usually accepted as an advantage of SIW structures, power handling capabilities of SIW filters has not been broadly studied yet from a multiphysics perspective considering electrical, thermal and mechanical effects at the same time.

In this work, the electric coupling structure proposed in [18] is studied in detail both for implementing direct and crosscouplings in combline SIW filters. As it is shown in the paper, this electric coupling scheme can be used to allow a further reduction of the filter size and increased bandwidth compared to conventional magnetic irises. Moreover, a fully packaged quasi-elliptic filter is designed, manufactured and measured. Finally, average and peak power handling capabilities of the device are theoretically studied and experimentally validated. The obtained results show that combline SIW filters can be a self-packaged, compact solution capable of incorporating both positive and negative couplings while keeping low-cost and batch-fabrication processes. The paper is structured as follows. In Section II, the negative coupling structure is presented and investigated. Section III provides the design, manufacturing and measurement of in-line three-pole combline SIW filters using the proposed negative coupling scheme. A comparison between magnetic and electric inter-resonator coupling is carried out, showing how the latter can be used to obtain more compact implementations while keeping the filter bandwidth. In Section IV, a fully packaged quasi-elliptic filter using a negative cross-coupling between non-adjacent resonators is presented, while the power handling capabilities of the filter are studied in Section $\mathrm{V}$ using a multiphysics approach to obtain the average and peak power capacities of the device. Finally, Section V presents the main conclusions.

\section{Negative Coupling Structure}

\section{A. Coaxial SIW resonator}

The building block resonator to be considered for the design of the in-line and quasi-elliptic bandpass filters is a combline resonator implemented in SIW technology [7]. The inductive section is obtained by a metallic post, implemented by a metallized via-hole, connected to ground at one of its ends. At the other side, a metallic disk having a radius $r_{p}$ much higher than the post diameter $d_{v}$ is connected. Between this disk (which can have a square or a circular shape) and the top ground plane a small air gap $s_{p}$ is inserted, so generating a high capacitance towards ground (termed loading capacitance $C_{l}$ ), which represents the capacitive section of the coaxial resonator. Fig. 1 shows the layout of a coaxial SIW resonator, including its main design parameters.

Such resonator can be modelled as a TEM-mode combline resonator embedded into the dielectric substrate. The TEMmode resonant frequency is given by the condition $B(\omega)=0$,

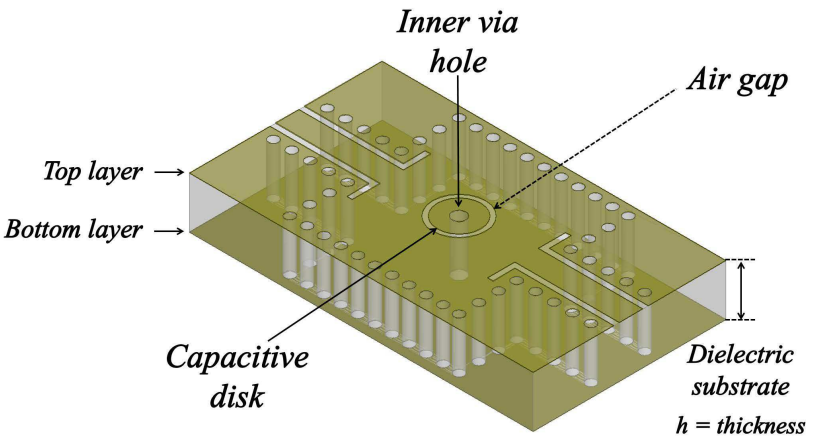

(a)

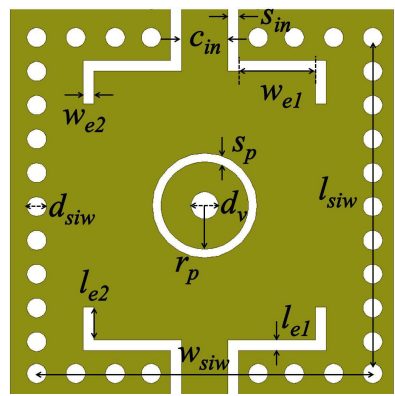

(b)

Fig. 1. (a) 3D view and (b) top view of the coaxial SIW resonator including its main design parameters.

where the susceptance of the coaxial SIW resonator can be expressed as

$$
B(\omega)=\omega C_{l}-\frac{1}{Z_{0}} \cot \beta h
$$

where $\beta$ is the propagation constant of the TEM-mode and $h$ the thickness of the substrate. Then, the characteristic impedance $Z_{0}$ of the coaxial resonator, and the ratio between the outer cavity side $l_{s i w}$ and the via diameter $d_{v}$ are obtained from [7]

$$
\begin{gathered}
Z_{0}=\left(\frac{\cot \theta_{0}+\csc ^{2} \theta_{0}}{2 b}\right) \\
\frac{l_{\text {siw }}}{d_{v}}=0.9268 \cdot e^{\frac{z_{0 \sqrt{\epsilon_{r}}}}{60}}
\end{gathered}
$$

where $\theta_{0}$ is the resonator electrical length at the design frequency $f_{0}$, that in our case is $\theta_{0}=\beta h$. The synthesis procedure starts by choosing the resonator slope parameter $b$ at the center angular frequency $\omega_{0}$ of the filter. The susceptance slope parameter $b$ for resonators having zero susceptance at $\omega_{0}$ is defined by [19]

$$
b=\left.\frac{\omega_{0}}{2} \frac{d B(\omega)}{d \omega}\right|_{\omega=\omega_{0}}=\frac{1}{2}\left[\omega_{0} C_{l}+\frac{\theta_{0}}{Z_{0} \sin ^{2} \theta_{0}}\right]
$$

The slope parameter has to be conveniently chosen as a trade-off between quality factor $Q$, compactness and physical feasibility of synthesized values of $C_{l}$ and $Z_{0}$. In order to increase component compactness, it is very important to set the inner via hole diameter to the minimum diameter allowed for the fabrication technology, as well as to keep $s_{p}$ as small as possible. This allows us to reduce radiation from apertures, thus helping to preserve a reasonable value for the resonator $Q$-factor. 


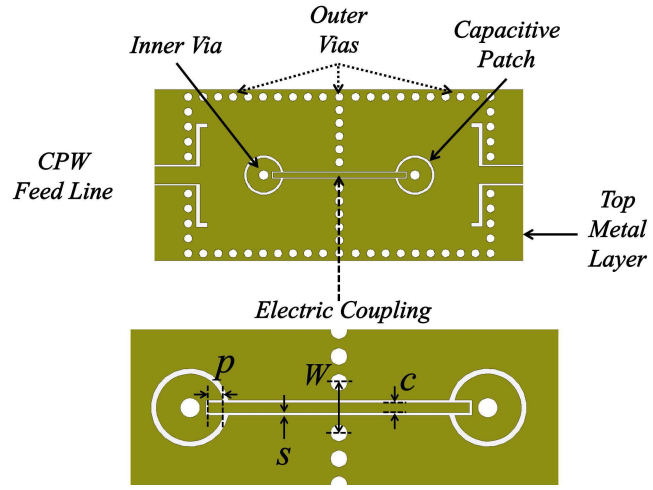

Fig. 2. Topology of the proposed electric coupling for coaxial SIW resonators.

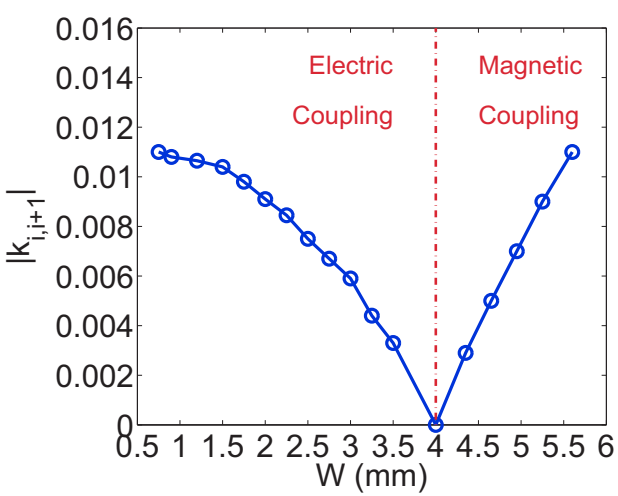

Fig. 3. Coupling coefficient $k_{i, i+1}$ variation versus iris width $W$ for $p=$ $1 \mathrm{~mm}, c=0.25 \mathrm{~mm}$ and $s=0.15 \mathrm{~mm}$. As the iris width increases, the total coupling decreases until it changes from electric to magnetic coupling and then increases again. In this figure, $l_{\text {siw }}=w_{\text {siw }}=10.6 \mathrm{~mm}$.

\section{B. Negative inter-resonator coupling}

The proposed solution to realize an electrical coupling between coaxial SIW resonators is based on a capacitive probe implemented by a high impedance coplanar line (CPW). The two ends of such a line penetrates into the head of the resonator capacitive patches, i.e. in the region with the highest intensity of electric field. A gap $s$ between the capacitive circular patch of the resonators and the probe is ensured. Part of the field is then collected by the probe and transferred to the adjacent resonator, so realizing an electric coupling.

In absence of the probe described above, the main source of coupling would be due to the magnetic field inside the SIW cavities. For the considered structure, the sign of the magnetic coupling is opposite to the electrical one. Therefore in order to boost the effect of the probe and get higher values of coupling, the magnetic coupling must be minimized. Such condition can be achieved with a wall of via holes placed across the resonators, which strongly confine the magnetic field coupling. The probe can still be realized if the width $c$ of the CPW line is sufficiently smaller than the gap $W$ between the central via-holes realizing the wall, as shown in Fig. 2.

Fig. 3 shows the total coupling $k_{i, i+1}$ vs. $W$ (obtained from the contribution of both electric and magnetic couplings), where $i$ is a generic $i$-th resonator. Such trace was derived with a constant value for the insertion $p$ (i.e. $p=1 \mathrm{~mm}$ ) of the

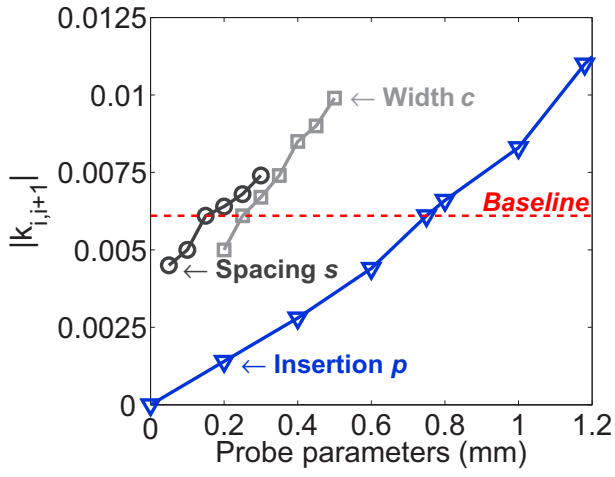

Fig. 4. Coupling coefficient $k_{i, i+1}$ variation versus CPW probe dimensions: conductor width $c$, spacing $s$ and insertion $p$ on capacitive disks. Red line is the simulation baseline (i.e. $s=0.15 \mathrm{~mm}, c=0.25 \mathrm{~mm}$ and $p=0.75 \mathrm{~mm}$ ). The post-wall iris width is $W=0.75 \mathrm{~mm}$.

probe inside the head of the resonator. For increasing values of $W$, the wall becomes more similar to a coupling iris with reference to classical waveguide structures. The curve shows that as the iris opens up, the total coupling starts to decrease until a certain point after which it starts to rise again.

This effect can be explained by considering that when $W$ is minimum, the iris is completely closed and we are in the condition of minimum magnetic coupling between the two resonators. Therefore, the major contribution to the coupling $k_{i, i+1}$ is given by the probe (i.e. electric coupling). When $W$ starts to increase, the magnetic coupling between the two resonators is not negligible anymore and it starts to counteract the effect of the electrical coupling, until they reach the same magnitude and the total coupling collapses to zero $\left(k_{i, i+1}=\right.$ $0)$. From this point on, if $W$ is increased the magnetic coupling starts to prevail, and the effect of the electric probe is not visible anymore. This analysis demonstrates that, in order to obtain electrical coupling between the two resonators, it is not sufficient to implement the probe described above, but it is also necessary to minimize the magnetic coupling by means of a post-wall iris. The insertion of the probe inside the head of the resonators is the main parameter to control the magnitude of the coupling $k_{i, i+1}$. The behaviour of $k_{i, i+1}$ vs. $p$ for the structure of Fig. 2 is shown in Fig. 4, which considers two resonators implemented on a substrate with $\epsilon_{r}=4.5 \pm 0.045$ and thickness $h=3.175 \mathrm{~mm}$. As expected, $k_{i, i+1}$ becomes stronger as the insertion $p$ within the head of the resonator is increased.

Clearly, $k_{i, i+1}$ also depends on $c$ and $s$. The behaviour of $k_{i, i+1}$ vs. $c$ and $s$ is also reported in Fig. 4. Nevertheless, the contribution of these parameters has a lower impact on the coupling magnitude. Thus, we can settle these values, and controlling the coupling by means of $p$.

A main advantage of the proposed electric coupling is to allow increasing direct inter-resonator coupling, especially for strongly loaded coaxial SIW resonators. As a way of comparison, the coupling strength capabilities of the magnetic and electric coupling schemes (Figs. 5 and 6) are compared. First, in Fig. 5, the coupling provided by a post-wall iris is depicted for two different values of slope parameter. As it is shown, by increasing the slope parameter of such structures, 


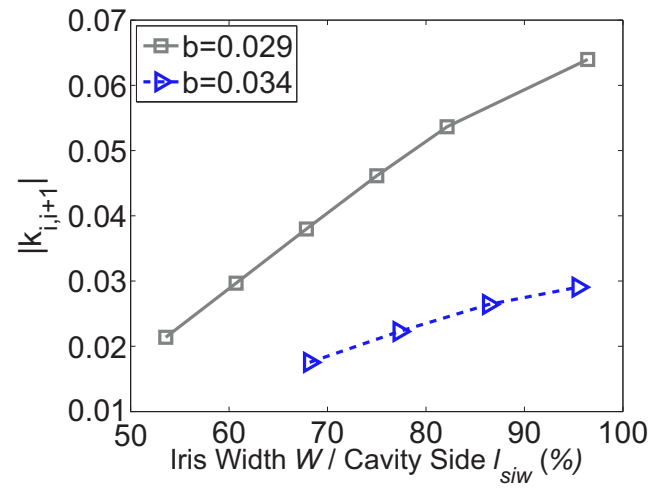

(a)

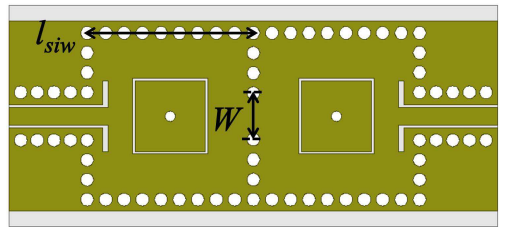

(b)

Fig. 5. (a) Coupling coefficient $k_{i, i+1}$ variation versus the relation between the post-wall iris width $W$ and the SIW cavity side $l_{\text {siw }}$. (b) Inter-resonator coupling system based on post-wall iris, showing design parameters.

the magnetic coupling is strongly reduced between coaxial SIW resonators. That variation of $b$, from 0.029 to 0.034 , corresponds to diminish the SIW cavity side from $15 \mathrm{~mm}$ to $11.6 \mathrm{~mm}$ while $C_{l}$ of the capacitive disks increases from $0.825 \mathrm{pF}$ to $0.95 \mathrm{pF}$. Both coaxial SIW resonators resonate in all cases at the same frequency, that is $5.5 \mathrm{GHz}$.

Now, by applying the proposed electric coupling for the same pair of coaxial resonators, it is possible to see how higher coupling values are easily implementable in more compact structures. Fig. 6 depicts the inter-resonator coupling values that can be obtained using the proposed electric CPW probe, and below the scheme of that solution is shown with its main design parameters.

It is worth mentioning that the insertion of the coupling probes modifies the value of $C_{l}$ of the capacitive patches, thus their sizes have to be slightly modified to meet specifications in terms of frequency. The proposed coupling scheme presents different advantages: single layer implementation, accurate control of the coupling level and it is well suited for tunable cross coupling using tuning elements, which can be easily mounted on the top metal layer.

\section{ThreE-Pole IN-LINE SIW FILTER WITH ElECTRIC COUPLING}

As it has been demonstrated in the previous Section II, the proposed electric coupling is an efficient approach for ensuring higher couplings between coaxial SIW resonators, and this is especially true when the resonator compactness has to be increased. The CPW probe used to obtain an electric coupling between coaxial SIW resonators can generate high values of coupling if the insertion of the probe inside the capacitive disks is opportunely chosen, independently of the SIW cavity size.

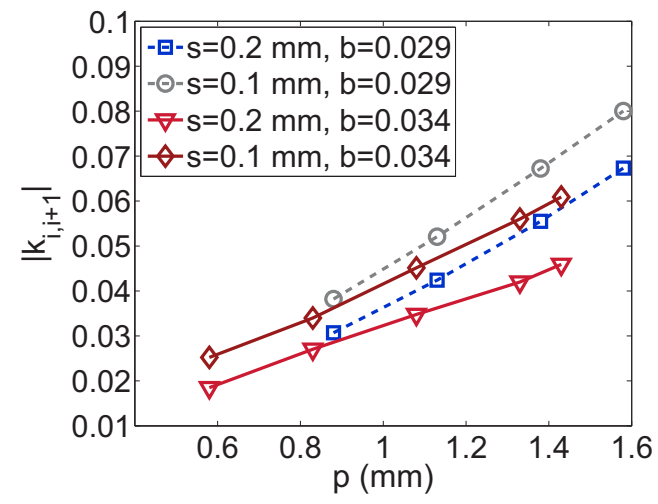

(a)

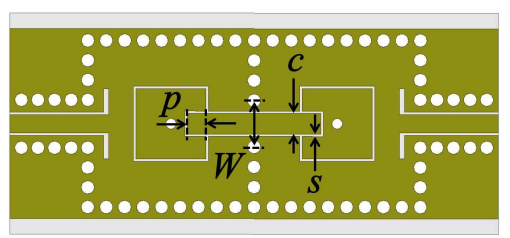

(b)

Fig. 6. (a) Coupling coefficient $k_{i, i+1}$ variation versus electric coupling probe insertion $p$. Other parameters are: $W=2.2 \mathrm{~mm}$ and $c=1.45 \mathrm{~mm}$. (b) Inter-resonator coupling system created at the top metal layer of the SIW resonators, showing design parameters.

Indeed, if the building block resonator is designed with a higher slope parameter $b$, the SIW cavity dimensions can be strongly reduced to compensate for the needed higher capacitive contribution of $C_{l}$ while maintaining the same resonant frequency, as deduced from (1)-(4). This circuit area reduction also leads to shift up the first spurious mode of the SIW cavity, widening the stopband bandwidth, so that, an improvement in terms of size and filter response can be really achieved.

In order to validate the aforementioned statements three third-order coaxial SIW filters having center frequency $5.5 \mathrm{GHz}$, passband bandwidth of $250 \mathrm{MHZ}$ (that corresponds to $4.6 \%$ ) and return losses better than $15 \mathrm{~dB}$ have been designed. The main difference between those filters shown in Fig. 7 is the coupling configuration used to couple resonators: filter in Fig. 7(a) presents magnetic couplings based on postwall iris, meanwhile filters in (b) and (c) are based on the proposed electric coupling. In particular, the filter shown in Fig. 7(c) is designed to present a higher resonator slope parameter $b$ (which leads to a small size cavity) in order to demonstrate the improved design characteristics added by using the electric coupling. It should be noted that this size reduction is incompatible with the use of the magnetic coupling scheme, since a smaller cavity leads to lower coupling values limiting the maximum achievable passband bandwidth. In this context, the electric coupling provides a higher design flexibility in order to fulfil requirements in terms of bandwidth, size and rejection band. Fig. 8 shows the coupling schemes of such three-pole coaxial SIW filters with magnetic and electric couplings.

The chosen dielectric substrate in these designs is now the 1.524 mm-thick Rogers R4003 with permittivity $\epsilon_{r}=$ 


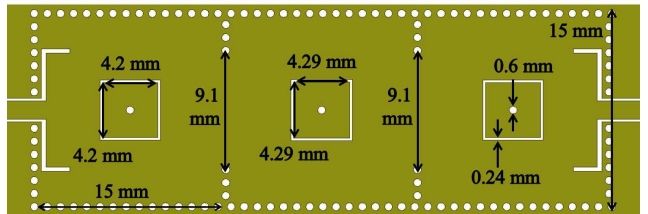

(a)

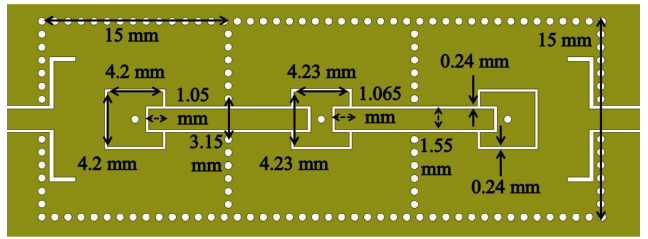

(b)

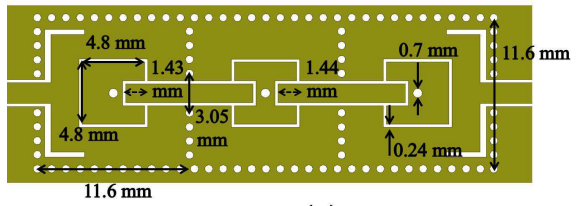

(c)

Fig. 7. Structure of the (a) magnetic-coupled, (b) electric-coupled and (c) compact electric-coupled coaxial SIW filters and main design dimensions.

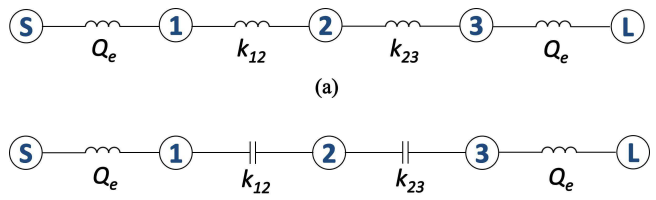

(b)

$$
k_{12}=k_{23}=0.0405 \quad Q_{e}=24.33
$$

Fig. 8. Multipath coupling diagram for the three-pole in-line coaxial SIW filter based on (a) magnetic (i.e. filter of Fig. 7(a)) and (b) electric coupling system (i.e. filters of Figs. 7(b)-(c)).

$3.55 \pm 0.05$. So, the resonator electrical length corresponds to $18.9^{\circ}$, i.e., $0.05 \lambda_{g}$, where $\lambda_{g}$ is the guided wavelength at $5.5 \mathrm{GHz}$. In the first two filters, the coaxial SIW resonators have been designed to present $b=0.029 \mathrm{~S}$ that gives a loading capacitance of $C_{l}=825 \mathrm{fF}$ while $Z_{0}=103.5 \Omega$. By using both values and choosing a diameter of $d_{v}=0.6 \mathrm{~mm}$ for the inner via hole, the SIW resonator cavity size is $w_{\text {siw }} \times l_{\text {siw }}=15 \times 15 \mathrm{~mm}^{2}$ (i.e. $0.52 \lambda_{g} \times 0.52 \lambda_{g}$ and $0.27 \lambda_{0} \times 0.27 \lambda_{0}$ ) whilst the square patch side is $4.2 \mathrm{~mm}$ with an air gap of $0.24 \mathrm{~mm}$ between the patch and the ground plane. The final structure of those coaxial SIW filters in Fig. 7(a) and (b) have a footprint of $15 \times 45 \mathrm{~mm}^{2}$, i.e. $0.52 \lambda_{g} \times 1.55 \lambda_{g}$ and $0.27 \lambda_{0} \times 0.82 \lambda_{0}$.

When the coaxial SIW resonator has $b=0.034 \mathrm{~S}, C_{l}$ and $Z_{0}$ become now $950 \mathrm{fF}$ and $89.4 \Omega$, respectively. The resonator cavity size consequently diminishes down to $w_{\text {siw }} \times l_{\text {siw }}=$ $11.6 \times 11.6 \mathrm{~mm}^{2}$, i.e. $0.4 \lambda_{g} \times 0.4 \lambda_{g}$ and $0.21 \lambda_{0} \times 0.21 \lambda_{0}$, having a square disk side of $4.8 \mathrm{~mm}$ and an air gap of $0.24 \mathrm{~mm}$. Finally, the compact electric coupling based filter shows a footprint of $11.6 \times 34.8 \mathrm{~mm}^{2}$, i.e. $0.4 \lambda_{g} \times 1.2 \lambda_{g}$ and $0.21 \lambda_{0} \times 0.63 \lambda_{0}$. The value of inter-resonator coupling for all filters are $k_{12}=k_{23}=0.0405$ while the external coupling coefficient is $Q_{e}=24.33$, as it is shown in Fig. 8.

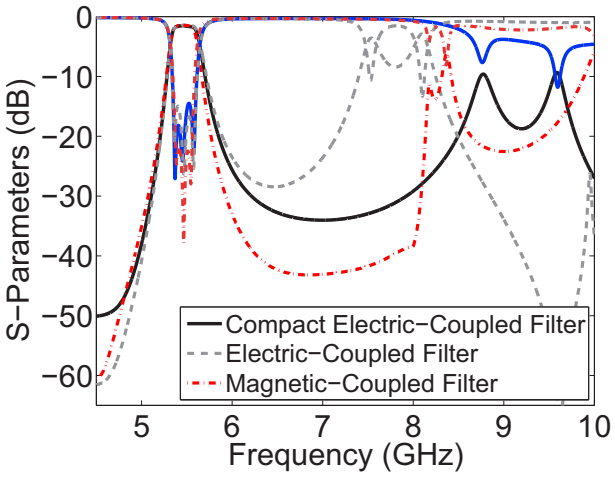

Fig. 9. Comparison of three simulated responses with same in-band characteristics but different stop-band behaviour.

Fig. 9 shows the simulation results of the three aforementioned filters. As it can be seen, the filter frequency responses above the passband show a higher order spurious band, which is due to the excitation of the $\mathrm{TE}_{101}$ mode of the SIW cavity. The best stopband performance is obtained by the filter of Fig. 7(c), as expected, with a wide stopband of up to $8.5 \mathrm{GHz}$ with more than $25 \mathrm{~dB}$ of rejection. In particular, this filter presents the same level of insertion losses ( $\mathrm{IL}=1.55 \mathrm{~dB}$ at $5.5 \mathrm{GHz}$ for all filters) with a $40 \%$ of total size reduction compared to the other two coaxial SIW filters of Figs. 7(a)(b) and a $70 \%$ of area reduction with respect to a standard $\mathrm{TE}_{101}$-mode SIW filter centered at $5.5 \mathrm{GHz}$. It should be noted that this passband bandwidth along with such a compact circuit area could not be implemented by using the magnetic coupling based on post-wall iris. The reason is that, as shown in Fig. 5, the maximum coupling values between two coaxial SIW resonators with slope parameters $b=0.034 \mathrm{~S}$ coupled by a magnetic coupling scheme results less than 0.03 , which is below the values needed for the third-order filter considered (see Fig. 8).

Table I gives the relation between unloaded quality factor $Q_{u}$ and miniaturization degree of a coaxial SIW resonator implemented in $1.524 \mathrm{~mm}$-thick Rogers R4003, when its slope parameter $b$ is increased. Those values have been compared to the area and $Q_{u}$ of a standard $\mathrm{TE}_{101}$ SIW cavity resonator centered at $5.5 \mathrm{GHz}$. As table I shows, the coaxial SIW topology allows high miniaturization of SIW structures with moderate degradation of $Q_{u}$.

TABLE I

$Q_{u}$ VERSUS MiNIATURIZATION FOR SIW CAVITY RESONATORS IMPLEMENTED IN ROGERS R4003 RESONATING AT 5.5 GHZ (UNIT: MM)

\begin{tabular}{|l|c|c|c|c|}
\hline SIW Topology & Area $\left(\mathrm{mm}^{2}\right)$ & $\Delta$ Area $(\%)$ & $Q_{u}$ & $\Delta Q_{u}(\%)$ \\
\hline TE $_{101}$ & 392 & 0 & 294 & 0 \\
\hline Coaxial $b=0.0265$ & 324 & -17 & 308 & +5 \\
\hline Coaxial $b=0.029$ & 213 & -46 & 274 & -7 \\
\hline Coaxial $b=0.034$ & 121 & -69 & 238 & -19 \\
\hline Coaxial $b=0.035$ & 90 & -77 & 230 & -22 \\
\hline
\end{tabular}

A photograph of the filter prototypes of Fig. 7(b) and (c) is shown in Fig. 10, while the simulated and measured responses of these filters are shown in Figs. 11 and 12, respectively. 


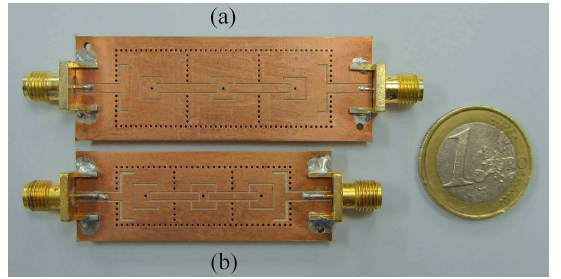

Fig. 10. Photography of the proposed three-pole in-line coaxial SIW filters based on electric coupling, where (a) $b=0.029 \mathrm{~S}$, and (b) $b=0.034 \mathrm{~S}$.

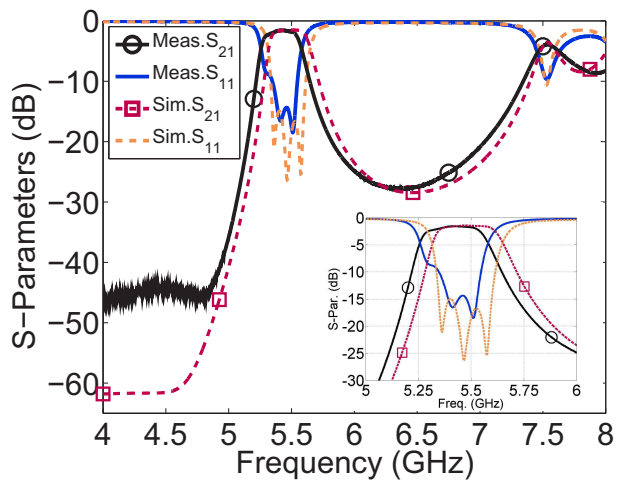

Fig. 11. Simulated (dashed line) and measured (solid line) wideband responses of the proposed three-pole in-line SIW filter with $b=0.029 \mathrm{~S}$ (Fig. 7(b)).

Measurements for both designs have validated the proposed concept, showing good insertion losses (i.e. $1.57 \mathrm{~dB}$ and $1.97 \mathrm{~dB}$ at $f_{0}$, respectively), and return losses better than $11 \mathrm{~dB}$ for both filters.

The measured response of filter prototype of Fig. 7(b) undergoes a frequency shift of $1.2 \%$ towards lower frequency, from $5.5 \mathrm{GHz}$ to $5.43 \mathrm{GHz}$ (see Fig. 11). For the compact three-pole filter of Fig. 7(c), the measured response has been shifted up to $5.9 \mathrm{GHz}$, as it can be observed in Fig. 12. These frequency deviations have been caused by an increase of the square patch air gap during fabrication process. The simulation results have taken into account such a variation in the air gap. Among others, the advantage of using such coupling scheme is that tuning devices can be opportunely used to create a reconfigurable filter response, which is not possible for an irisbased magnetic coupling.

\section{Four-Pole Cross-Coupled SIW Filter}

\section{A. Filter Design}

In order to further demonstrate the proposed coupling solution, its application on a 4-pole narrow-band filter with a quasielliptic frequency response is now considered. The coupling scheme of the filter is given in Fig. 13. The coupling between resonators 1 and 4 , termed $k_{14}$, has opposite sign compared to all the inter-resonator couplings. This configuration is particularly interesting because it improves the selectivity generating TZs located both above and below the filter passband. The filter center frequency is chosen to be $5.75 \mathrm{GHz}$ with an equiripple fractional bandwidth FBW of $2 \%$ (114 MHZ), which results in a simulated $1-\mathrm{dB}$ bandwidth of $94 \mathrm{MHZ}(\mathrm{FBW}=$ 1.64\%). The TZs are set at 5.63 and $5.87 \mathrm{GHz}$, respectively,

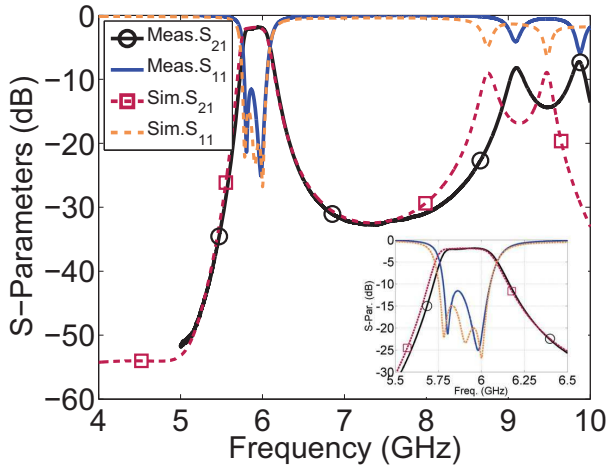

Fig. 12. Simulated (dashed line) and measured (solid line) wideband responses of the proposed three-pole in-line coaxial SIW filter with $b=0.034 \mathrm{~S}$ (Fig. 7(c)).

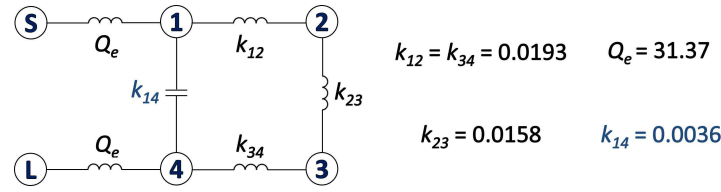

Fig. 13. Multipath coupling diagram for the cross-coupled filter.

corresponding to the center frequencies of adjacent filters in a multiplexer application with contiguous channels.

In this design, the slope parameter of the combline SIW resonator is chosen to be $b=0.016 \mathrm{~S}$, while the substrate presents $\epsilon_{r}=4.5$ and thickness $h=3.175 \mathrm{~mm}$. As it was previously mentioned, the resonator electrical length corresponding to substrate thickness $h$ is $46.5^{\circ}$, i.e., $0.13 \lambda_{g}$, where $\lambda_{g}$ is the guided wavelength at $5.75 \mathrm{GHz}$. This gives a loading capacitance of $C_{l}=33.5 \mathrm{fF}$ while the characteristic coaxial impedance is $Z_{0}=78.35 \Omega$. By taking both previous values and choosing a diameter of $d_{v}=0.7 \mathrm{~mm}$ for the metallic via hole implementing the coaxial topology, the resonator structure can be optimized with EM full-wave simulations, by adjusting the size of the loading capacitive disk and the gap that separates it from the top metal layer. The resonators size is $w_{\text {siw }} \times l_{\text {siw }}=10.6 \times 10.6 \mathrm{~mm}^{2}$ (i.e. $0.43 \lambda_{g} \times 0.43 \lambda_{g}$ ) whilst the capacitive disk radius is $0.915 \mathrm{~mm}$ with an air gap of $0.15 \mathrm{~mm}$ between the disk and the ground plane. The final structure of the filter presents a very compact footprint of $21.2 \times 21.2 \mathrm{~mm}^{2}\left(0.86 \lambda_{g} \times 0.86 \lambda_{g}\right)$, which corresponds to $63 \%$ of area reduction compared to a quasi-elliptic four-pole filter based on $\mathrm{TE}_{101}$-mode SIW resonators and implemented in the same dielectric substrate.

The input-output coupling is realized by means of coplanar waveguide-to-SIW transition with $90^{\circ}$ bend slots, which are etched from the metal on top of the first (last) cavity, see Fig. 14. The value of the $Q_{e}$ can be easily controlled by modifying the dimensions of the probe. The coupling coefficients are $k_{12}=k_{34}=0.0193, k_{23}=0.0158, k_{14}=-0.0036$ and $Q_{e}=31.37$.

Fig. 14 shows the geometric configuration of the 4-pole cross-coupled filter, including the proposed electric crosscoupling and some main filter dimensions. The coupling between the SIW resonators 1 and 4 and the input/output ports 


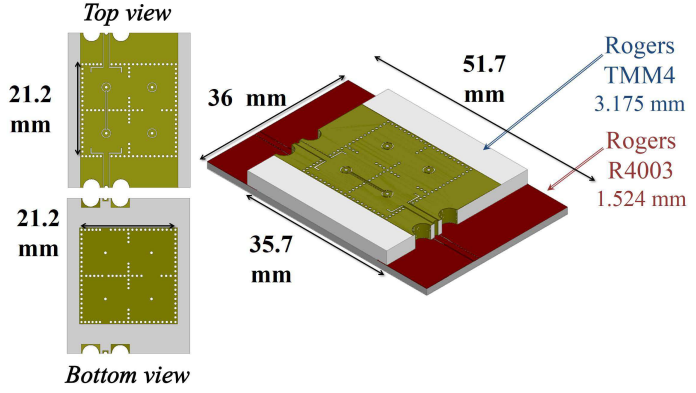

Fig. 14. Layout of the cross-coupled SIW filter assembled on a carrier substrate. Open-ended CPW line provides the electric coupling.

are realized by means of CPW current probes that are etched on the top metal layer. The $Q_{\text {ext }}$ decreases with increasing size of ground plane openings that are created at the end of the CPW line. The filter dimensions are given in Table II. From simulations, the minimum insertion loss is $2.2 \mathrm{~dB}$ and the two TZs are located at 5.63 and $5.87 \mathrm{GHz}$ providing $35 \mathrm{~dB}$ and $27 \mathrm{~dB}$ of minimum rejection, respectively.

TABLE II

LAYOUT Dimensions of THE DESIGNED FiLTER (Unit: MM)

\begin{tabular}{|l|l||l|l||l|l||l|l|}
\hline$d_{\text {siw }}$ & 0.6 & $W$ & 0.85 & $w_{e 1}$ & 2.6 & $r_{p 1}$ & 0.915 \\
\hline$l_{\text {siw }}$ & 10.6 & $s$ & 0.15 & $w_{e 2}$ & 0.3 & $r_{p 2}$ & 0.943 \\
\hline$w_{\text {siw }}$ & 10.6 & $c$ & 0.25 & $l_{e 1}$ & 0.3 & $r_{p 3}$ & 0.943 \\
\hline$d_{v}$ & 0.7 & $p$ & 0.32 & $l_{e 2}$ & 1.08 & $r_{p 4}$ & 0.915 \\
\hline$c_{\text {in }}$ & 1.2 & $s_{\text {in }}$ & 0.15 & $s_{p}$ & 0.2 & $h$ & 3.175 \\
\hline
\end{tabular}

\section{B. Vertical Transition}

In Fig. 14, below the SIW filter substrate, it can be seen a host board with two isolated coplanar lines which were implemented on a 1.524 mm-thick Rogers R4003. In fact, the considered four-pole filter has been designed for allowing surface mounting device (SMD) assembly considering a PCB fabrication process. In order to enable SMD packaging of the filter, connection must be provided to the filter input and output. This kind of self-packaged solution for coaxial SIW filters, which provides access to the input/output ports through castellated plated via holes, was originally proposed in [20] showing promising results. This solution offers potential advantages in terms of design flexibility, low loss and enabling the SMD integration of the device.

The proposed system is illustrated in Fig. 15, where a scheme of the vertical transition structure is shown. The bottom layer will interface between the carrier board and the packaged filter through a vertical pseudo-CPW structure. Since the CPW structure has both signal and ground on the same plane, the connection of the device to the host board is simple. In fact, the device can be easily placed and soldered onto the carrier substrate. The diameter and spacing of the transition half-a-hole vias are the most important design parameters for controlling the EM performance [20].

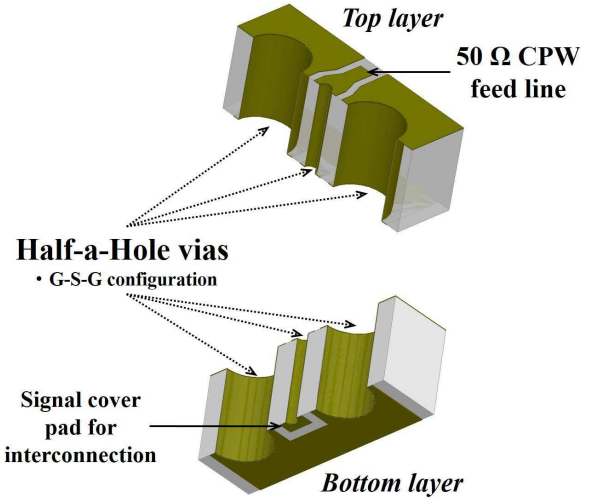

Fig. 15. Side views of the vertical CPW-CPW transition that allows SMD assembly of the packaged filter on a carrier substrate.

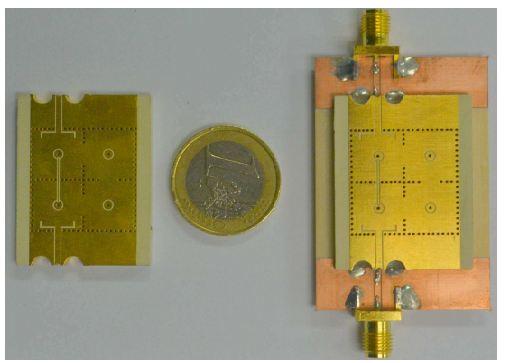

Fig. 16. Photography of the filter prototype assembled on its substrate carrier.

\section{Experimental Results}

The designed filter has been fabricated on a $3.175 \mathrm{~mm}$-thick Rogers TMM4 substrate $\left(\epsilon_{r}=4.5 \pm 0.045, \tan \delta=2 \cdot 10^{-3}\right)$ using a standard single-side PCB process. The diameter and center-to-center pitch of the external via holes are $0.6 \mathrm{~mm}$ and $1.1 \mathrm{~mm}$, respectively. A photograph of the filter prototype is shown in Fig. 16 while Fig. 17 shows the frequency responses of the cross-coupled SIW filter. It is worth mentioning that simulated and measured $S$-parameters include the vertical transition for connecting input/output ports. The measured 1$\mathrm{dB}$ bandwidth is $102.5 \mathrm{MHZ}$ that corresponds to $1.8 \%$, while the bandwidth at $-20 \mathrm{~dB}$ corresponds to $200 \mathrm{MHZ}$. The Sparameters of the fabricated filter prototype were measured with an Agilent E8364B PNA series network analyzer, and a TRL calibration was performed using a home-made CPW cal-kit.

The frequency shift of $70 \mathrm{MHZ}(\approx 1.2 \%)$ between simulations and results is due to the fabrication tolerances which, however, can be corrected in the filter design process. The minimum IL has increased up to $3.6 \mathrm{~dB}$ due to fabrication issues of the vertical transitions, resulting in an estimated Qfactor of 225 , being 320 the simulated resonator $Q_{u}$. The measured return losses are better than $25 \mathrm{~dB}$. The upper stopband is in very good agreement with simulations up to $12 \mathrm{GHz}$, being the attenuation always better than $30 \mathrm{~dB}$, as shown in Fig. 17. Table III shows the comparison between this work and other cross-coupled SIW filters shown in the references, where it can be observed the high compactness degree obtained with the proposed solution. 


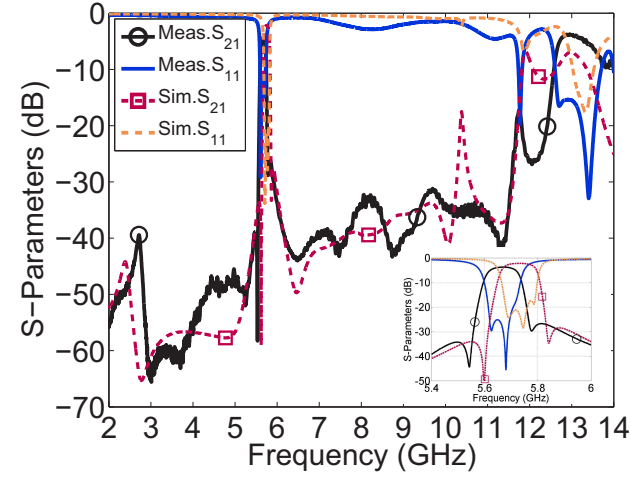

Fig. 17. Simulated (dashed line) and measured (solid line) wideband responses of the proposed SIW filter.

TABLE III

COMPARISON OF CROSS-COUPLED FILTERS IN SIW TECH. (Note: B = BELOW, $\mathrm{A}=\mathrm{ABOVE})$

\begin{tabular}{|c|c|c|c|c|c|}
\hline & $f_{0}(\mathrm{GHz})$ & FBW & $\mathrm{IL}(\mathrm{dB})$ & $\operatorname{Size}\left(\lambda_{g}^{2}\right)$ & $\mathrm{TZs}$ \\
\hline$[12]$ & 20.5 & $4 \%$ & 0.9 & $1.42 \times 1.36$ & $1 \mathrm{~B}, 1 \mathrm{~A}$ \\
\hline$[14]$ & 27 & $7.4 \%$ & 2.1 & $1.45 \times 1.14$ & $1 \mathrm{~B}, 2 \mathrm{~A}$ \\
\hline$[21]$ & 2.5 & $4 \%$ & $\approx 4$ & $1.6 \times 1.6$ & $1 \mathrm{~B}, 1 \mathrm{~A}$ \\
\hline$[16]$ & 5.1 & $4.2 \%$ & 2.05 & $1.55 \times 0.98$ & $1 \mathrm{~B}, 1 \mathrm{~A}$ \\
\hline$[17]$ & 20 & $3 \%$ & 1.7 & $1.88 \times 1.42$ & $2 \mathrm{~B}, 2 \mathrm{~A}$ \\
\hline This work & 5.75 & $1.8 \%$ & 3.6 & $0.86 \times 0.86$ & $1 \mathrm{~B}, 1 \mathrm{~A}$ \\
\hline
\end{tabular}

\section{Multiphysics Study For THE POWER HANDLing CAPABILITY EVALUATION OF SIW COMBLINE FILTERS}

In this section the power handling capability (PHC) of the SIW combline resonator is studied in detail. Two different kind of studies are addressed: a) Average Power Handling Capability (APHC), where it is assumed that a CW signal is applied to the circuit and the electro-thermo-mechanical coupling is analyzed in order to determine the maximum temperature and thermal stress of the circuit as a function of the input signal power. And b) Peak Power Handling Capability (PPHC), in such a case pulsed signals are applied to the circuit and the corona discharges are examined as a function of the input signal power and pressure. As an example of analysis, the PHC study is focused on the quasi-elliptic filter presented in Section IV.

\section{A. Average Power Handling Capability}

For moderated $\mathrm{CW}$ signal powers (1-5 W) high temperatures can be achieved in planar circuits due to self-heating, which limits the APHC [22]-[24]. Planar circuits present three loss mechanisms: conductive, dielectric and radiation losses. They are linearly proportional to the input power, but only the two former loss mechanisms produce heat in the circuit, so that they can be defined as the internal heat sources of the structure. In particular, dielectric loss is treated as a volumetric heat source whereas conductive loss as a surface heat source. In pure planar technologies as microstrip, the gradient of temperature due to self-heating is computed by determining the conductor and dielectric losses, then, the heat flow distribution in the microstrip cross section is derived to finally obtain

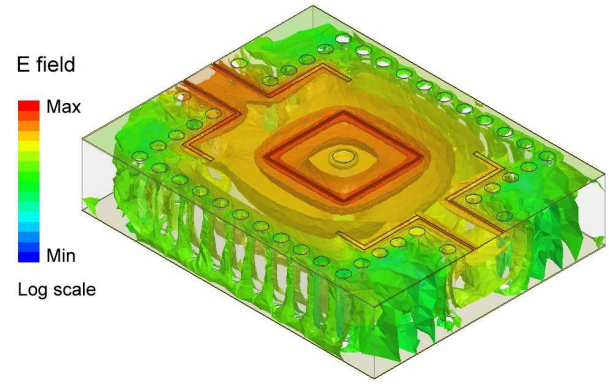

Fig. 18. 3D Electric Field distribution for a SIW Combline cavity.

the temperature rise that defines the maximum working input power [22], [23], [25]. In SIW technology, due to its large aspect ratio $w / h$, and the fact that conductive losses are equal both in top and bottom planes, the whole heat source $Q_{\text {int }}$ can be assumed to be homogeneous in a differential region $w \cdot h \cdot l$, consequently providing a homogeneous temperature in this volumetric region. This assumption is also favoured by the use of via holes conforming the SIW, which propagate the heat between the top and bottom layers. From the Newton's Law of cooling, the generated heat is equal to the total heat delivered to the environment by means of convection and/or thermal radiation. Thus, this energy balance can be written as

$$
\begin{aligned}
Q_{i n t}+Q_{e x t}= & \sum_{i=1}^{m} h_{c o n v, i} A_{i}\left(T_{h o t}-T_{a m b}\right) \\
& +\sum_{j=1}^{n} h_{r a d, j} A_{j}\left(T_{h o t}-T_{a m b}\right)
\end{aligned}
$$

where

$$
Q_{i n t}=\Gamma P_{i n}
$$

being $\Gamma$ the loss factor of the structure and $P_{i n}$ the input signal power. $Q_{\text {ext }}$ is any external heat source (such as solar radiation), $T_{a m b}$ is the temperature in the surroundings, $A_{i}$ and $A_{j}$ are the surface layers exposed to convection and radiation boundary conditions, respectively, and $h_{\text {conv }}$ and $h_{\text {rad }}$ are the convection and radiation coefficients. The values $h_{\text {conv }}$ and $h_{\text {rad }}$ depend on the external conditions of each layer, i.e., if there is a heat sink attached, natural or forced convection, emissivity, etc. From (5), the maximum temperature $T_{\text {hot }}$ can be computed for a given $P_{i n}$ and if the environment conditions $\left(h_{c o n v}\right.$ and $\left.h_{\text {rad }}\right)$ are known. An expected conclusion from this equation is that, if $Q_{\text {int }}$ is kept, the bigger the surface $A$, the lower the $T_{h o t}$ is, and therefore, the higher the APHC.

For the SIW combline resonators, the electric-magnetic field distribution which defines the heat source pattern is different to that presented by standard SIW rectangular cavities, as seen in Fig. 18. However, the large aspect ratio $w / h$ as well as the use of the outer via holes and the inner conductor, make the temperature in the SIW combline cavity nearly homogeneous, and therefore, (5) can be used as a first order approximation.

For the APHC analysis of the filter, firstly, the power loss per resonator is computed from the equivalent lumped element circuit whose behaviour is modeled by the coupling matrix shown in Fig. 13. $Q_{u}=320, f_{0}=5.75 \mathrm{GHz}$ and FBW $=2 \%$ 


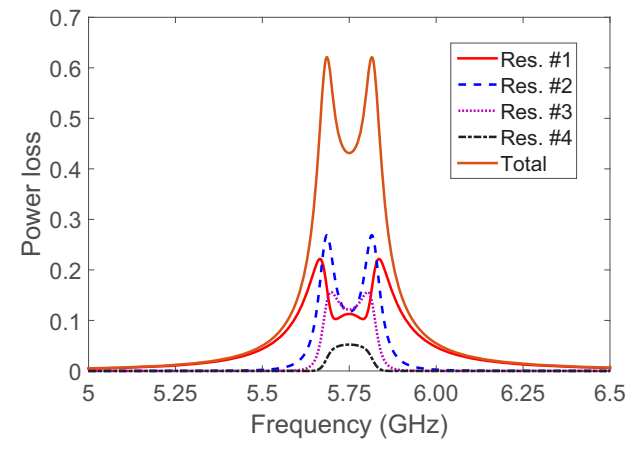

Fig. 19. Power loss in each resonator per watt from the equivalent lumped element circuit model. $Q_{u}=320$.

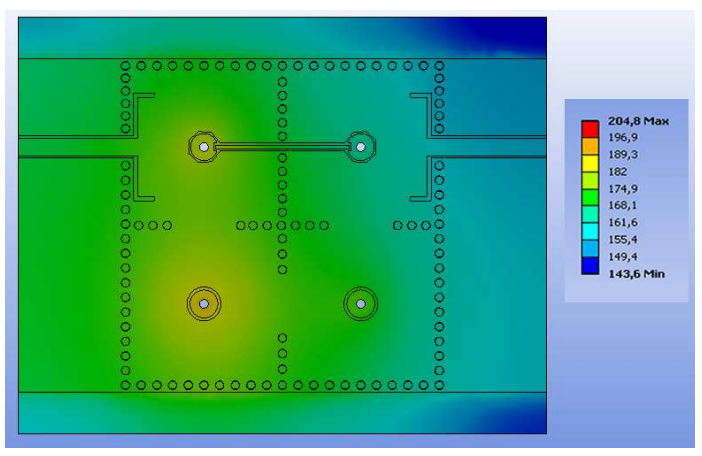

Fig. 20. Thermal profile of the filter presented in Section IV. $P_{i n}=5 \mathrm{~W}$ at $5.82 \mathrm{GHz}$ with natural convection around the circuit with $h_{\text {conv }}=$ $10 \mathrm{~W} / \mathrm{m}^{2} \cdot{ }^{\circ} \mathrm{C}$ and $T_{a m b}=22{ }^{\circ} \mathrm{C}$.

are used for such a computation. As seen from Fig. 19, the total highest power loss is found at the inband corners, at 5.68 and $5.82 \mathrm{GHz}$, with $\Gamma=0.62$. In particular, resonator \#2 presents the highest level of losses, so that it is expected that this resonator limits the APHC. (5) can be used in order to calculate the average temperature in the whole circuit. For $P_{\text {in }}=5 \mathrm{~W}$ at $5.82 \mathrm{GHz}$, assuming natural convection with $h_{\text {conv }}=10 \mathrm{~W} / \mathrm{m}^{2} \cdot{ }^{\circ} \mathrm{C}$ and $T_{a m b}=22{ }^{\circ} \mathrm{C}$, with a whole circuit area of $A_{\text {top }}=A_{\text {bottom }}=35.7 \times 28.2 \mathrm{~mm}^{2}$ and neglecting thermal radiation, the average temperature in the circuit is found to be $176{ }^{\circ} \mathrm{C}$. ANSYS Multiphysics is used in order to accurately obtain the thermal profile for such an example. Fig. 20 shows its thermal profile where the hottest spot is found in resonator $\# 2$, as predicted, with a value of $205{ }^{\circ} \mathrm{C}$. The simulated average temperature in the circuit is very close to the computed value according to (5).

The APHC is limited by that $P_{i n}$ which creates such a thermal gradient leading to exceed the glass transition temperature of the substrate $T_{g}$ or, that $P_{i n}$ which generates a thermal stress able to destroy (or deform) the circuit. The minimum of those values limits the APHC. For the electro-thermo-mechanical coupling needed to evaluate the thermal stress, ANSYS Multiphysics is used again. Table IV summarizes the thermomechanical parameters required for the study. It should be remarked that the thermal conductivity in the substrate both in SIW and SIW coaxial cavities is not a critical parameter for the reduction of the gradient of temperature as it is in microstrip technology. The yield strength point gives the maximum stress
TABLE IV

THERMO-MECHANICAL PROPERTIES OF TMM 4

\begin{tabular}{c|c|c}
\hline \hline & Copper & Dielectric \\
\hline Thermal conductivity $\left(\mathrm{W} / \mathrm{m}^{\circ}{ }^{\circ} \mathrm{C}\right)$ & 400 & 0.7 \\
CTE $(\mathrm{x}, \mathrm{y}, \mathrm{z})\left(\mathrm{ppm} /{ }^{\circ} \mathrm{C}\right)$ & 17 & $16,16,21$ \\
Young's Modulus $(\mathrm{GPa})$ & 117 & 11.9 \\
Poisson's Ratio & 0.33 & 0.2 \\
Yield Strength $(\mathrm{MPa})$ & 220 & - \\
Glass Transition Temperature $\left({ }^{\circ} \mathrm{C}\right)$ & - & 425
\end{tabular}

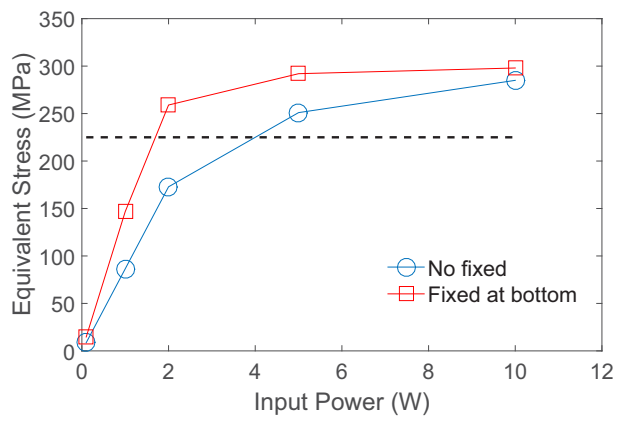

Fig. 21. Maximum equivalent Von-Mises Stress as a function of $P_{i n}$. At $5.82 \mathrm{GHz}$ and assuming natural convection on the circuit with $T_{a m b}=22{ }^{\circ} \mathrm{C}$.

that a material can afford before permanent deformation. It defines the transition between the linear mechanical behaviour (elastic behaviour) of the material and the non-linear one.

Fig. 21 shows the maximum equivalent stress (Von-Mises) as a function of $P_{i n}$ at the frequency where losses are the highest $(5.82 \mathrm{GHz})$ and for two different load cases (bottom of the circuit is fixed or unfixed). Obviously, when the bottom is fixed the stress produced is higher and the elastic limit is reached for lower power levels. The maximum stress happens in the metal, both in layers and the via holes, whereas the stress provoked in the substrate is much lower. From these figures, it can be concluded that $P_{\text {in }}<2 \mathrm{~W}$ in order to keep the circuit in safety ranges of applied power. It should be noted that for this circuit, due to the high value of $T_{g}$, APHC is limited by thermal stress. With respect to the deformation produced in the circuit while the $\mathrm{CW}$ signal is applied, in the case of the bottom is fixed, deformation is insignificant (maximum deformation $<8 \mu \mathrm{m}$ for $P_{i n}=5 \mathrm{~W}$ ). In the case when the bottom is unfixed, although the maximum deformation is still low, it can produce a small frequency shift in the transfer function for high $P_{i n}$ (maximum deformation $<82 \mu \mathrm{m}$ for $\left.P_{\text {in }}=5 \mathrm{~W}\right)$.

\section{B. Peak Power Handling Capability}

The SIW combline resonator presents an important density of electric field through the air, between the loading capacitive disk and the top layer ground. Thus, this resonator can be susceptible to present corona discharges for high power applied signals. The situation is different for standard SIW technology, since the electric field is mainly distributed through the dielectric and the critical areas for air ionization are the slot edges of the coplanar line feeding the SIW cavities [1]. 


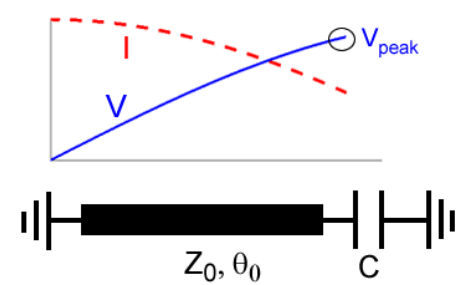

Fig. 22. Scheme of the SIW combline resonator where the current and voltage distributions are shown at resonance frequency.

In order to evaluate any possible corona discharge, the maximum voltage (and consequently, the expected maximum electric field) of the SIW combline filter should be found as a function of the input signal power. From the equivalent lumped element circuit, the voltage and current in each resonator $i$ of the filter can be computed as

$$
\begin{gathered}
v_{i}=\frac{v_{g}}{q_{e}}[A]_{i, 1}^{-1} \\
i_{i}=\frac{v_{g}}{q_{e} R}[A]_{i, 1}^{-1}
\end{gathered}
$$

where $v_{g}$ is the generator voltage, $q_{e}$ the normalized external quality factor, $R$ is the reference impedance and $[A]$ is the normalized impedance/admittance matrix defining the filter network [19]. Once the equivalent voltages/currents are known, the stored energy can be found as

$$
W_{i}=\frac{4 P_{i n}}{q_{e} \mathrm{BW}}[A]_{i, 1}^{-2}
$$

where $P_{i n}$ is the available power at the input port and BW is the absolute bandwidth of the filter in rad/s. At resonance, the stored energy by every resonator of the equivalent lumped element network should be the same as that associated to the distributed resonators [26], [27]. Fig. 22 shows the scheme of a combline resonator where the voltage and current standing waves are also plotted at resonance. From the standing waves the stored energy by the distributed resonator $W_{d i s t r, i}$ can be calculated as [28], [29] by integrating the standing waves (in form of sinusoidal functions) along the resonator. Thus, by making $W_{i}=W_{d i s t r, i}$ the peak voltage in the SIW combline resonator can be found at the capacitive patch as

$$
V_{\text {peak }, i}=4 \sqrt{\frac{P_{i n} Z_{0, i}}{q_{e} \mathrm{FBW} \chi}}[A]_{i, 1}^{-1}
$$

where

$$
\chi=\theta_{0}+\frac{\sin 2 \theta_{0}}{2}
$$

It should be noted that for the case $\theta_{0}$ is small, the SIW combline resonator behaves as a lumped element resonator, and therefore, the values found with (10) are the same as those obtained with (7).

The air ionization is a phenomenon linked to the electric field strength rather than to the voltage [27], [30]. So, the electric field strength must be estimated in the capacitive patch of the resonators forming the filter. Although the electric field distribution along the air gap is not evident, as a fast approximation the maximum electric field in each resonator can be computed as

$$
\left|E_{\text {peak }, i}\right|=\frac{V_{\text {peak }, i}}{s_{i}}
$$

where $s_{i}$ is the annular gap of the resonator $i$. For high pressure regime (pressures $>100 \mathrm{mbar}$ ), the air ionization breakdown threshold (peak value) can be determined by following the rule [31]

$$
\left|E_{\text {break }}\right|=42.7 \sqrt{p^{2}+2 f^{2}}(\mathrm{~V} / \mathrm{cm})
$$

where $p$ is the pressure in torr and $f$ is the operation frequency in GHz. At this point, from (12) and (13) the PPHC can be analytically computed in filters based on combline resonators for high pressure regime. For low pressure regime, the continuity equation describing the electron density evolution must be solved. This arduous task must be done numerically, in this work the software tool AURORASAT ${ }^{\mathrm{TM}}$ SPARK3D ${ }^{\circledR}$ is employed. This tool uses the real electromagnetic field distribution of the device under test in order to solve the continuity equation, and provides the power breakdown threshold of the device from some input parameters such as pressure, kind of gas (air or nitrogen) or temperature. As a validation example, the PPHC is computed for the quasi-elliptic filter by using the equations derived in the previous lines and by using SPARK3D. The input parameters are $f=5.82 \mathrm{GHz}$ (where the voltage is maximum, which happens at the capacitive patch of resonator \#2; this frequency coincides with the frequency of maximum losses) and $p=500$ mbar. From the derived equations, PPHC is analytically calculated as $7.3 \mathrm{~W}$ whereas from SPARK3D is $11.2 \mathrm{~W}$. If the maximum electric field strength of the structure is simply taken from an electromagnetic tool (such as HFSS or CST; note that a normalization could be needed depending on the software used), and by applying the rule (13), the obtained PPHC is $0.9 \mathrm{~W}$, which is a value considerably lower than those previously obtained. From these results, it can be concluded that taking the maximum electric field from the EM simulation results, which is indeed a strategy commonly used for waveguides or coaxial resonators, gives a very conservative limit for PPHC in SIW combline resonators. This is due to the fact that the maximum electric field values are very concentrated around the capacitive patch edges, in a region involving just some microns, which is not enough to alter the electron density. Additionally, some field singularities could appear in the resolution of the field around such corner edges. Another conclusion is that the approximation made in (12) gives a reasonable value of electric field strength in order to determine the PPHC.

Fig. 23 shows the Paschen curves of the filter for each resonator, obtained from SPARK3D at the frequency where losses and voltage magnification are maximum. Resonator \#2 is limiting the PPHC, as expected. The critical pressure is around 5-10 mbar, where PPHC is just $0.6 \mathrm{~W}$. So, it is presumed that in a low pressure regime corona limits PHC rather than the thermo-mechanical effects. An interesting effect has been also detected in this filtering structure: due to the sharp shape of the first (and fourth) resonator corners (see Fig. 24), it is found that there is a high concentration of electric 


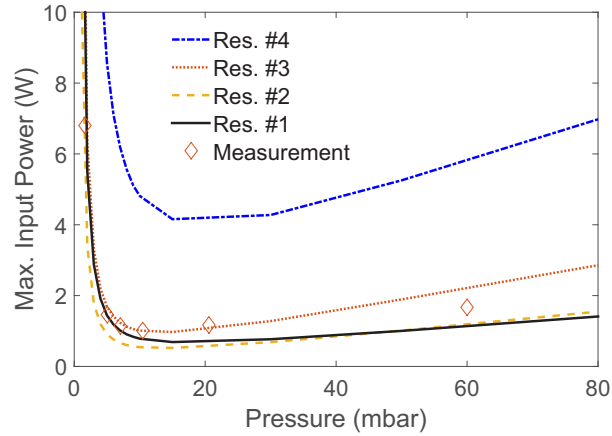

(a)

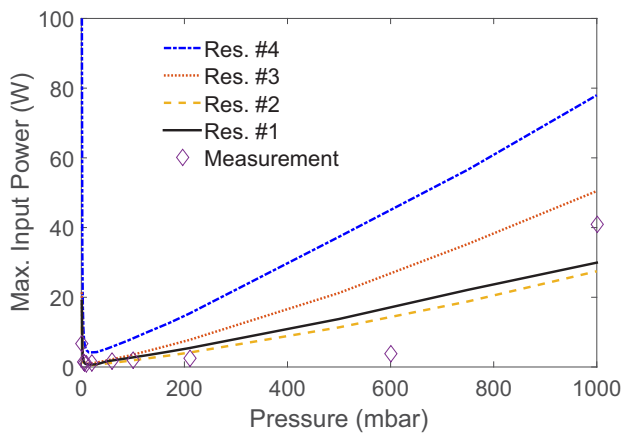

(b)

Fig. 23. Simulated Paschen Curves for the resonators involved in the filter under test. (a) Critical pressure region. (b) The whole pressure range. In this simulation, $f=5.82 \mathrm{GHz}$ and $T_{a m b}=22{ }^{\circ} \mathrm{C}$.

field density around those corners, providing higher values of electric field strength than those of resonator \#2. Thus, resonator \#1 may limit the PPHC of the filter even though the voltage in this resonator is lower than that in resonator \#2 at the frequency of analysis. However, after fabrication, those corners are rounded, as seen in Fig. 24(b), leading to a reduction of the electric field density around them. Fig. 24(c) shows the simulated Paschen Curves for the first resonator when the corners are sharped and rounded. As deduced from this figure, rounding the corners can considerably increase PPHC in this filter topology. In Fig. 23 the Paschen Curve of resonator \#1 corresponds to the rounded corner case. Obviously, for both cases the filter response remains constant.

\section{APHC and PPHC Measurements}

In order to validate the calculated and simulated results, high power measurements have been performed at the European High-Power RF Space Laboratory (Valencia, Spain). Fig. 25 shows a schematic diagram of the employed test-bed. Several methods have been used for the corona discharge detection: third harmonic detection, nulling of the forward/reverse power at the operation frequency, electron probe and by visual inspection by recording the circuit with a video camera. The applied signal to the filter has been a pulsed signal with a carrier frequency of $5.75 \mathrm{GHz}$ (frequency where the voltage magnification and losses are maximum in the measured filter), a pulse width of $20 \mu \mathrm{s}$ and a duty cycle of $1 \%$. These pulsed signal characteristics avoid any self-heating effect in the device, whereas the pulse width is wide enough to assume

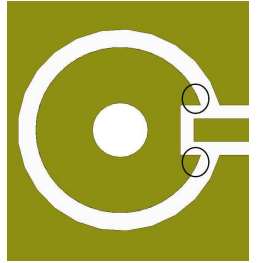

(a)

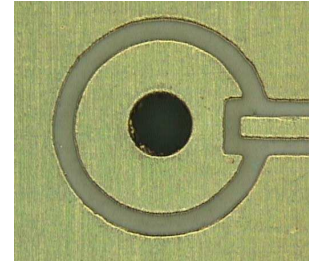

(b)

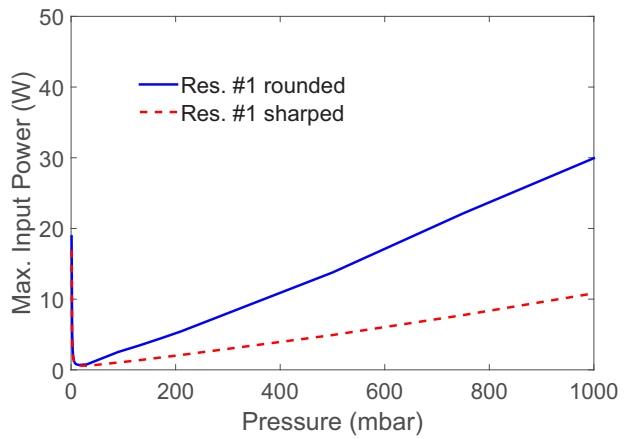

(c)
Fig. 24. Zoom in of the resonator \#1. (a) Layout. (b) Fabricated. (c) Simulated Paschen Curves comparison between the sharp and rounded corner cases.

that the pulse breakdown threshold converges to the $\mathrm{CW}$ one. The PPHC has been evaluated for different pressures in order to obtain the Paschen Curves of the device.

In Fig. 23 the measured data can be also observed. For all scenarios corona breakdown has firstly appeared in resonator $\# 2$, as expected. There is a reasonable good agreement (especially at lower pressures) with the simulated results what validates the theoretical prediction and the study done. The maximum difference between the measured and simulated breakdown field levels has been of $45 \%$, which has been found for a pressure of 600 mbar. Fig. 26 shows the capture from the video camera at the moment of a corona discharge has occurred. As seen, the spark is uniformly originated along the annular slot.

With respect to the $\mathrm{APHC}$ measurement, a $\mathrm{CW}$ signal at the same frequency has been applied to the circuit at ambient pressure ( $p=1000$ mbar) and temperature. The temperature has been measured by means of thermocouples attached to the resonators in regions where their impact is low. The circuit was suspended so that natural convection can be assumed on all faces. The thermal steady-state was reached after around 5 minutes the signal is switched on. The measured temperatures for the different $\mathrm{CW}$ applied signal power samples have been close to the analytically calculated and simulated values, with differences lower than 20\%. Up to $5 \mathrm{~W}$ there were no evidences of any rupture point in the circuit, however $P_{i n}>5 \mathrm{~W}$ should be avoided since temperatures higher than $150{ }^{\circ} \mathrm{C}$ were measured. Furthermore, for $P_{i n}>5 \mathrm{~W}$ a frequency shift started being noticed in the measured response as well as a considerable increase of losses. After switching on/off the applied signal, the circuit could recover its original electromagnetic performance, although probably with a slight permanent material deformation. The $\mathrm{CW}$ applied power was increased up to obtain the complete 


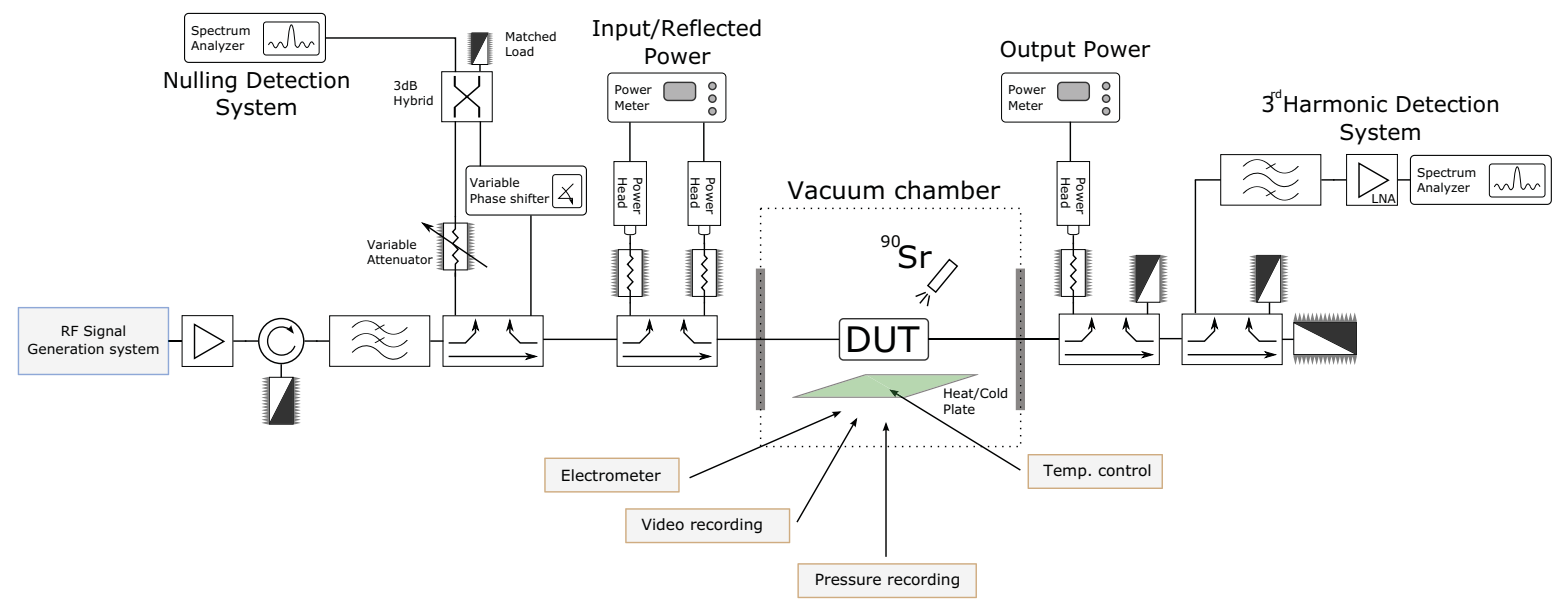

Fig. 25. Scheme of the testbed configuration used for corona breakdown detection.

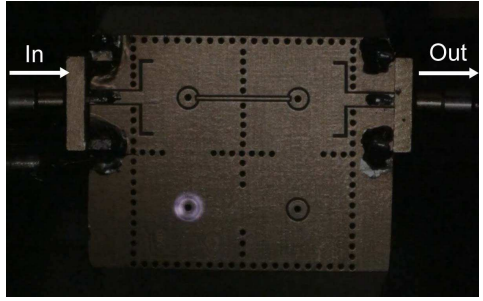

Fig. 26. Capture of a corona discharge at the second resonator of the filter under test.

failure of the device. The circuit was finally destroyed by a continuous corona discharge which occurred for $P_{\text {in }}=14 \mathrm{~W}$. This value is lower than the one measured at ambient pressure for the pulsed signal case and shown in Fig. 23. This can be due to the following reasons: the air temperature was much higher because of self-heating, which reduces the corona breakdown threshold for high pressure regime and, because corona breakdown thresholds are normally lower for the $\mathrm{CW}$ case than for the pulsed signal case, unless a very wide pulsed signal width is used.

\section{CONCLUSions}

In this paper the design of SIW combline resonator filters with advanced performances has been investigated. For this aim, different coupling configurations involving magnetic and electric coupling mechanisms have been proposed and studied in detail. It has been demonstrated that the proposed electric coupling configuration provides a high flexibility in filter design, which allows us to design very compact filters by keeping a reasonable level of losses, as well as to increase the maximum achievable passband bandwidth in SIW coaxial technology. Additionally, it has been proposed such a filter where the magnetic and electric coupling schemes has been arranged in order to design a very compact quasi-elliptic narrowband bandpass filter, presenting a very selective response with a wide rejection band. The power handling capability (both average and peak) has been also studied for such filtering structures from a multiphysics point of view. As it has been shown, these filters can afford moderate levels of power in spite of their small circuit area and narrow band. All concepts have been validated by the simulation and measurement of some fabricated proof-of-concept filtering devices. High power measurements have been also carried on, which have verified the proposed multiphysics study.

\section{ACKNOWLEDGMENTS}

The authors would like to thank Marta Reglero and Máriam Taroncher for their valuable help and discussions with the realization of the power handling measurements. Our acknowledgment also to Val Space Consortium for its contribution Laboratories funded by the European Regional Development Fund - A way of making Europe. We would like also to thank the "Lab-STICC Computing Platform" at the University of Brest (France) for their support in order to perform the multiphysics simulations.

\section{REFERENCES}

[1] X.-P. Chen and K. Wu, "Substrate integrated waveguide filters: Practical aspects and design considerations," IEEE Microwave Mag., vol. 15, no. 7, pp. 75-83, Nov. 2014.

[2] N. Grigoropoulos, B. Sanz-Izquiredo, and P. Young, "Substrate integrated folded waveguides (sifw) and filters," IEEE Microw. Wireless Compon. Lett., vol. 15, no. 12, pp. 829-831, Dec. 2005.

[3] Y. Wang, W. Hong, Y. Dong, B. Lui, H. Tang, J. Chen, X. Yin, and $\mathrm{K}$. Wu, "Half mode substrate integrated waveguide (hmsiw) bandpass filter," IEEE Microw. Wireless Compon. Lett., vol. 17, no. 4, pp. 265267, Apr. 2007.

[4] C. Jin and Z. Shen, "Compact triple-mode filter based on quarter-mode substrate integrated waveguide," IEEE Trans. Microwave Theory Tech., vol. 62 , no. 1, pp. 37-45, Jan. 2014

[5] Y. D. Dong, T. Yang, and T. Itoh, "Substrate integrated waveguide loaded by complementary split-ring resonators and its applications to miniaturized waveguide filters," IEEE Trans. Microwave Theory Tech., vol. 57, no. 9, pp. 2211-2223, Sep. 2009.

[6] L.-S. Wu, L. Zhou, X.-L. Zhou, and W.-Y. Yin, "Bandpass filter using substrate integrated waveguide cavity loaded with dielectric rod," IEEE Microw. Wireless Compon. Lett., vol. 19, no. 8, pp. 491-493, Aug. 2009.

[7] J. D. Martínez, S. Sirci, M. Taroncher, and V. E. Boria, "Compact CPWFed combline filter in Substrate Integrated Waveguide technology," IEEE Microw. Wireless Compon. Lett., vol. 22, no. 1, pp. 7-9, Jan. 2012.

[8] R. Levy, "Filters with single transmission zeros at real or imaginary frequencies," IEEE Trans. Microwave Theory Tech., vol. MTT-24, no. 4, pp. 172-181, Apr. 1976. 
[9] J. Thomas, "Cross-coupling in coaxial cavity filters-A tutorial overview," IEEE Trans. Microwave Theory Tech., vol. 51, no. 4, pp. 1368-1376, Apr. 2003.

[10] X. Chen, W. Hong, T. Cui, J. Chen, and K. Wu, "Substrate integrated waveguide (SIW) linear phase filter," IEEE Microw. Wireless Compon. Lett., vol. 15, no. 11, pp. 787-789, Nov. 2005.

[11] J. D. Martínez, S. Sirci, and V. E. Boria, "Compact substrate integrated waveguide filter with asymmetric frequency response for c-band wireless applications," in IEEE MTT-S Int. Wir. Symp. (IWS), Beijing, China, Apr 2013, pp. $1-4$

[12] X. P. Chen and K. Wu, "Substrate Integrated Waveguide cross-coupled filter with negative coupling structure," IEEE Trans. Microwave Theory Techn., vol. 56, no. 1, pp. 142-149, Jan. 2008.

[13] G. H. Lee, C. S. Yoo, J. G. Yook, and J. C. Kim, "Siw quasi-elliptic filter based on LTCC for 60-GHz application," in 4th Eur. Microw. Integ. Circuits Conf., Sep. 2009, pp. 204-207.

[14] K. Gong, W. Hong, Y. Zhang, P. Chen, and C. J. You, "Substrate integrated waveguide quasi-elliptic filters with controllable electric and magnetic mixed coupling," IEEE Trans. Microwave Theory Techn., vol. 60, no. 10, pp. 3071-3078, Oct. 2012.

[15] B. Potelon, J. Favennec, C. Quendo, E. Rius, C. Person, and J. Bohorquez, "Design of a substrate integrated waveguide (SIW) filter using a novel topology of coupling," IEEE Microw. Wireless Compon. Lett., vol. 18 , no. 9, pp. 596-598, Sep. 2008.

[16] W. Shen, W. Y. Yin, X. W. Sun, and L. S. Wu, "Substrate-Integrated Waveguide bandpass filters with planar resonators for System-onPackage," IEEE Trans. Compon. Packag. Manufact. Technol., vol. 3, no. 2, pp. 253-261, Feb. 2013

[17] F. Zhu, W. Hong, J. X. Chen, and K. Wu, "Cross-coupled Substrate Integrated Waveguide filters with improved stopband performance," IEEE Microw. Wireless Compon. Lett., vol. 22, no. 12, pp. 633-635, Dec. 2012.

[18] S. Sirci, F. Gentili, J. D. Martínez, V. Boria, and R. Sorrentino, "Quasielliptic filer based on SIW combline resonators using a coplanar line cross-coupling," in IEEE MTT-S Int. Microw. Symp. Dig., Phoenix, USA, May 2015 , pp. $1-4$

[19] J. S. Hong, Microstrip Filter for RF/Microwave Applications, 2nd ed. Hoboken, NJ, USA: John Wiley and Sons, Inc., Feb. 2011.

[20] S. Sirci, J. Martínez, R. Stefanini, P. Blondy, and V. Boria, "Compact SMD packaged tunable filter based on substrate integrated coaxial resonators," in IEEE MTT-S Int. Microw. Symp. Dig., Tampa Bay, USA, Jun 2014, pp. 1-4.

[21] J. Lee, E. J. Naglich, H. Sigmarsson, D. Peroulis, and W. J. Chappell, "Tunable Inter-Resonator coupling structure with positive and negative values and its application to the Field-Programmable Filter Array (FPFA)," IEEE Trans. Microwave Theory Techn., vol. 59, no. 12, pp. 3389-3400, Dec. 2011

[22] K. C. Gupta, R. Garg, I. J. Bahl, and P. Bhartia, Microstrip lines and slotlines, 2nd ed. Artech house Boston, 1996.

[23] M. Sanchez-Soriano, Y. Quere, V. Le Saux, C. Quendo, and S. Cadiou, "Average power handling capability of microstrip passive circuits considering metal housing and environment conditions," IEEE Trans. Compon., Packag., Manufact. Technol., vol. 4, no. 10, pp. 1624-1633, Oct. 2014.

[24] M. Sanchez-Soriano, M. Edwards, Y. Quere, D. Andersson, S. Cadiou, and C. Quendo, "Mutiphysics study of rf/microwave planar devices: Effect of the input signal power," in 15th EuroSime, Apr. 2014, pp. 1-7.

[25] I. Bahl and K. Gupta, "Average power-handling capability of microstrip lines," IEE J. Microw. Opt. Acoust. UK, vol. 3, no. 1, pp. 1-4, 1979.

[26] C. Ernst and V. Postoyalko, "Prediction of peak internal fields in directcoupled-cavity filters," IEEE Trans. Microwave Theory Tech., vol. 51, no. 1, pp. 64-73, Jan. 2003.

[27] M. Yu, "Power-handling capability for rf filters," IEEE Microwave Mag., vol. 8, no. 5, pp. 88-97, Oct. 2007.

[28] R. E. Collin, Foundations for microwave engineering. John Wiley \& Sons, 2007.

[29] M. Sánchez-Soriano, E. Bronchalo, and G. Torregrosa-Penalva, "Parallel-coupled line filter design from an energetic coupling approach," IET Microw., Antennas, Propag., vol. 5, no. 5, pp. 568-575, Apr. 2011

[30] R. J. Cameron, C. M. Kudsia, and R. R. Mansour, Microwave filters for communication systems. Wiley-Interscience, 2007.

[31] W. Woo and J. DeGroot, "Microwave absorption and plasma heating due to microwave breakdown in the atmosphere," Phys. Fluids, vol. 27, no. 2, pp. 475-487, 1984.

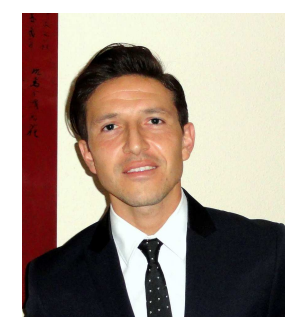

Stefano Sirci (S'14) received the B.S. and M.S degrees (with distinction) in Electronic Engineering from the University of Perugia, Perugia, Italy, in 2006 and 2009, respectively. In 2009, he carried out his Master thesis on efficient modal computation in arbitrarily shaped waveguides by BI-RME Method with the Polytechnic University of Valencia, Valencia, Spain. He is current working toward the $\mathrm{PhD}$ degree at the Polytechnic University of Valencia. From 2010 to 2015, he has been with the Microwave Application Group (GAM) at the Institute of Telecommunications and Multimedia Applications (iTEAM) at the Polytechnic University of Valencia. His working is focused on emerging technologies for reconfigurable microwave components with emphasis on designing, fabrication and measurement of tunable microwave SIW filters, in PCB and LTCC technologies.

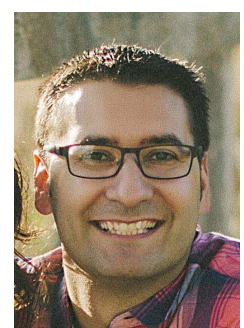

Miguel Ángel Sánchez-Soriano (S'09-M'13) was born in Yecla (Murcia), Spain, in 1984. He received the Telecommunications Engineer degree (with a Special Award) and the Ph.D degree on Electrical Engineering from the Miguel Hernandez University (UMH), Spain, in 2007 and 2012, respectively. In 2007 he joined the Radiofrequency Systems Group $\mathrm{UMH}$, as a research assistant. He was a visiting researcher with the Microwaves Group headed by Prof. Jia-Sheng Hong at Heriot-Watt University, Edinburgh, UK, in 2010. In January 2013 he joined the LabSTICC group (Université de Bretagne Occidentale, Brest, France) as a Postdoctoral Researcher, where he worked for 2 years. Since January 2015 , he is a "Juan de la Cierva" research fellow at the "Grupo de Aplicaciones de Microondas" (GAM) (Polytechnic University of Valencia, Spain). His research interests cover the analysis and design of microwave planar devices, especially filters and their reconfigurability, and the multiphysics study of high frequency devices.

Dr. Sánchez-Soriano was the recipient of the runner-up HISPASAT award to the Best Spanish Doctoral Thesis in New Applications for Satellite Communications, awarded by the Spanish Telecommunication Engineers Association (COIT/AEIT), and the Extraordinary Ph.D award from the Miguel Hernndez University. He serves as a reviewer for various journals and conferences, including the IEEE Transactions on Microwaves, Theory and Techniques, the IEEE Microwave and Wireless Components Letters and the IET Microwaves, Antennas and Propagation.

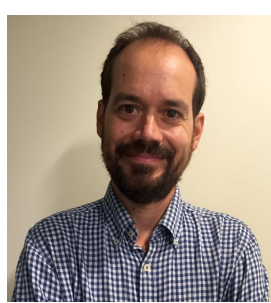

Jorge D. Martínez was born in Murcia, Spain, on February 15, 1979, and he is Member of the IEEE, $\mathrm{PhD}$, and Associate Professor at the School of Telecommunication Engineering of the Polytechnic University of Valencia (UPV) since 2012. Previously, he received his degree in Telecommunication Engineering and the $\mathrm{PhD}$ degree from the same institution (UPV), in 2002 and 2008, respectively. He joined the Department of Electronics Engineering of the UPV in 2002 as a research fellow, and he became Assistant Professor in 2009. During 2007 he was a Research Visitor at XLIM, CNRS and University of Limoges (France), where he worked on the design and fabrication of RF MEMS components under the advice of Prof. Pierre Blondy. He is a researcher of the I3M R\&D institute at UPV, where he actively collaborates with the Microwave Applications Group (GAM). At I3M premises, he is now the Technical Responsible of the Laboratory for High Frequency Circuits Fabrication (LCAF) of the UPV, focused on Low Temperature Co-fired Ceramics (LTCC) and other related multi-layered technologies. His current research interests are focused on emerging technologies for reconfigurable microwave components with emphasis on tuneable filters and RF MEMS, and the design and fabrication of advanced microwave filters in planar and substrate integrated waveguide technologies, as well as the application of multi-layer fabrication technologies to $\mathrm{RF} /$ microwave and millimetre-wave applications. 


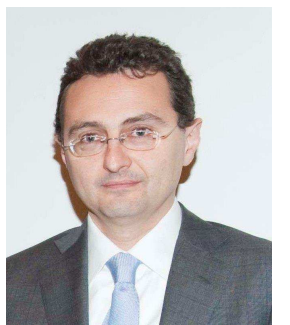

Vicente E. Boria (S'91-A'99-SM'02) was born in Valencia, Spain, on May 18, 1970. He received his Ingeniero de Telecomunicacin degree (with firstclass honors) and the Doctor Ingeniero de Telecomunicacin degree from the Polytechnic University of Valencia, Valencia, Spain, in 1993 and 1997, respectively. In 1993 he joined the Departamento de Comunicaciones, Polytechnic University of Valencia, where he has been Full Professor since 2003. In 1995 and 1996, he was holding a Spanish Trainee position with the European Space Research and Technology Centre, European Space Agency (ESTEC-ESA), Noordwijk, The Netherlands, where he was involved in the area of EM analysis and design of passive waveguide devices. He has authored or co-authored 10 chapters in technical textbooks, 125 papers in refereed international technical journals, and over 175 papers in international conference proceedings. His current research interests are focused on the analysis and automated design of passive components, left-handed and periodic structures, as well as on the simulation and measurement of power effects in passive waveguide systems.

Dr. Boria has been a member of the IEEE Microwave Theory and Techniques Society (IEEE MTT-S) and the IEEE Antennas and Propagation Society (IEEE AP-S) since 1992. He is member of the Editorial Boards of the IEEE Transactions on Microwave Theory and Techniques, IEEE Microwave and Wireless Components Letters, Proceeding of the IET (Microwaves, Antennas and Propagation), IET Electronics Letters and Radio Science. Since 2013 , he serves as Associate Editor of IEEE Microwave and Wireless Components Letters. He is also a member of the Technical Committees of the IEEEMTT International Microwave Symposium and of the European Microwave Conference.

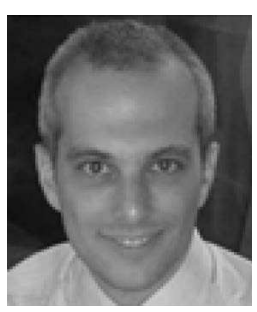

Fabrizio Gentili (GSM'12-A'14) was born in Foligno, Italy, in 1984. He received the Master Laurea degree (with distinction) in electronic engineering and Ph.D. degree from the University of Perugia, Perugia, Italy, in 2010 and 2014, respectively. His doctoral dissertation concerned innovative solutions for RF and microwave filters. In 2009, he carried out his Master thesis on photonic nanoantennas with the University of Bristol, Bristol, U.K. In March 2010, he joined RF Microtech, where his research concerned the design of antennas and passive microwave components. Since April 2014, he has been a Post-Doctoral Researcher with the Institute of Microwave and Photonic Engineering, Graz University of Technology, Graz, Austria.

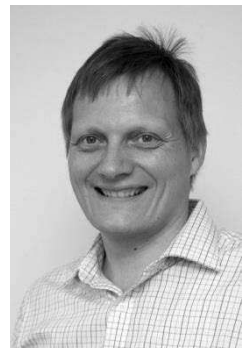

Wolfgang Bösch (F13) received his engineering degrees from the Technical University of Vienna and Graz in Austria. He finalized his MBA with distinction at Bradford University School of Management, U.K., in 2004. He joined the Graz University of Technology, Austria, in March 2010 to establish a new Institute for Microwave and Photonic Engineering. Previously he was the CTO of the Advanced Digital Institute in the U.K., a not for profit organization to promote research activities. He has also been the Director of Business and Technology Integration of RFMD UK. For almost 10 years he has been with Filtronic plc as CTO of Filtronic Integrated Products and Director of the Global Technology Group. Prior to joining Filtronic, he held positions in the European Space Agency (ESA) working on amplifier linearization techniques, MPR-Teltech in Canada working on MMIC technology projects and the Corporate R\&D group of M/A-COM in Boston, MA, USA, where he worked on advanced topologies for high efficiency power amplifiers. For four years he was with Daimler-Chrysler Aerospace in Germany, working on T/R Modules for airborne radar. Dr. Bösch is a Fellow of the IET. He has published more than 80 papers and holds 4 patents. He was a Non-Executive Director of Diamond Microwave Devices (DMD) and the Advanced Digital Institute (ADI). Currently he is a Non-Executive Director of VIPER-RF (U.K.).

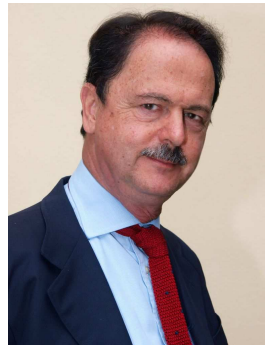

Roberto Sorrentino (LF'90) is a Professor at University of Perugia, Perugia, Italy, where he was the Chairman of the Electronic Department, Director of the Computer Center (1990-1995), and Dean of the Faculty of Engineering (1995-2001). His research activities have been concerned with various technical subjects, but mainly with numerical methods and CAD techniques for passive microwave structures and the analysis and design of microwave and millimetre-wave circuits including filters and antennas. In recent years he has been involved in the modelling and design of Radio Frequency Microelectromechanical Systems (RF-MEMS) and their applications on tuneable and reconfigurable circuits and antennas. He is the author or co-author of more than 150 technical papers in international journals and 200 refereed conference papers. He has edited a book for IEEE Press (Numerical Methods for Passive Microwave Structures, 1989) and co-authored four books: Advanced Modal Analysis (with M. Guglielmi and G. Conciauro), J. Wiley, 2000; RF and Microwave Engineering (in Italian) (with G. Bianchi) McGrawHill, 2006; Electronic Filter Simulation and Design (with G. Bianchi) McGrawHill, 2007, RF and Microwave Engineering, (with G. Bianchi) J. Wiley, 2010. In 1990 he has become a Fellow of the IEEE for contribution to the modelling of planar and quasi-planar microwave and millimetre-wave circuits. He has received several international awards and recognitions: in 1993 the IEEE MTT-S Meritorious Service Award, in 2000 the IEEE Third Millennium Medal, in 2004 the Distinguished Educator Award from IEEE MTT-S, in 2010 the Distinguished Service Award from the European Microwave Association, in 2012, together with S. Bastioli and C. Tomassoni, the Microwave prize for the paper A New Class of Waveguide Dual-Mode Filters Using TM and Nonresonating Modes. In 2015 he was awarded the Microwave Career Award from the IEEE MTTSociety.

Roberto Sorrentino has been active within the IEEE MTT Society. From 1984 through 1987 he was the Chairman of the IEEE Section of Central and South Italy and was the founder of the local MTT/AP Chapter that he chaired from 1984 to 1987. From Jan. 1995 through April 1998 he was the Editorin-Chief of the IEEE Microwave and Guided Wave Letters. From 1998 to 2005 he has served on the Administrative Committee of the IEEE Microwave Theory and Techniques Society. He was elected again in MTT AdCom for the term 2011-2013. He is also a member of Technical Committees MTT-15 on Field Theory and MTT-1 on Computer-Aided Design, which he chaired in 2003-04. Roberto Sorrentino served the International Union of Radio Science (URSI) as Vice Chair (1993-1996) then Chair (1996-1999) of the Commission D (Electronics and Photonics). Since 2007 he is the President of the Italian Commission of URSI. In 2002 he was among the founders and first President of the Italian Electromagnetic Society (SIEm) that he chaired until 2008. From 1998 to 2005 he was a member of the High Technical Council of the Italian Ministry of Communications. In 1998 he was one of the founders of the European Microwave Association (EuMA) and was its President till 2009. In 2007 he founded RF Microtech, a spin-off company of the University if Perugia dealing with RF MEMS, microwave systems and antennas. 